\title{
ABELIAN COVERS AND ISOTRIVIAL CANONICAL FIBRATIONS
}

\author{
Francesco Zucconi \\ Dipartimento di Matematica e Informatica \\ Università di Udine*
}

\begin{abstract}
We give a pure algebraic method to construct all the infinite families of surfaces $S$ with isotrivial canonical fibration where $S$ is the minimal desingularization of $X=Z / G$ and $G$ is an Abelian group acting diagonally on the product of two smooth curves: $Z=F \times D$. In particular we recover all the known infinite families of surfaces with isotrivial canonical fibration and we produce many new ones. Our method works in every dimension and, with minor modifications, it can be applied to construct surfaces with canonical map of degree $>1$.
\end{abstract}

\section{Introduction.}

An infinite family $\mathcal{F}=\left\{S_{t}\right\}_{t \in T}$ of surfaces is a set which intersects an infinite number of irreducible components of the moduli space of all surfaces of general type: that is, for every natural number $N \in \mathbb{Z}^{+}$there exists $t \in T$ such that $\chi\left(K_{S_{t}}\right)>N$, where $K_{S_{t}}$ is the canonical sheaf, $\chi\left(K_{S_{t}}\right)=1-q\left(S_{t}\right)+p_{g}\left(S_{t}\right), p_{g}\left(S_{t}\right)=\operatorname{dim}_{\mathbb{C}} H^{0}\left(S_{t}, K_{S_{t}}\right)$, $q\left(S_{t}\right)=\operatorname{dim}_{\mathbb{C}} H^{1}\left(S_{t}, K_{S_{t}}\right)$.

After Beauville [3] showed that for some families of surfaces with $p_{g} \gg 0$ the image $B$ of the canonical map $\Phi_{\left|K_{S}\right|}: S-\rightarrow \mathbb{P}\left(H^{0}\left(S, K_{S}\right)^{\star}\right)$ is a curve, it was worthwhile taking the trouble to establish a geography of these surfaces; other authors [16], [17], [9], [15], [10], [19] developed the project. It was known soon that: $a$ ) if $B$ is a curve and $\chi\left(K_{S}\right) \geq 21$ then $\Phi_{\left|K_{S}\right|}$ is a morphism and $2 \leq g \leq 5$ where $g$ is the genus of the fibre, see [3] [proposition 2.1]; b) $B$ is the rational normal curve of degree $p_{g}-1$ or the elliptic normal curve of degree $p_{g}$ and $0 \leq q(S) \leq 2$, [17]. So far some infinite families of surfaces with canonical fibration $\phi_{\left|K_{S}\right|}: S \rightarrow B$ of genus $g=2$ or $g=3$ have been found; they split into two cases: $i) \phi_{\left|K_{S}\right|}: S \rightarrow B$ is an isotrivial fibration, that is, the smooth fibres are isomorphic each other; $i i$ ) it is not an isotrivial fibration; for this second class we refer to [6] where the reader can find a complete bibliography.

*Research carried out under the project "Geometria Algebrica, Algebra Commutativa e aspetti comutazionali" (coordinatore nazionale Claudio Pedrini). 
The purpose of this article is to present a uniform and algebraic procedure to construct infinite families of surfaces with isotrivial canonical fibration; see Theorem 2.3. Moreover we will show that the case $g=5$ does not occur as Xiao conjectured in 18 and we will construct explicitly all the genus $\leq 3$ isotrivial canonical fibrations $f: S \rightarrow B$ where $S$ is the minimal desingularization of $X=Z / G$ and $G$ is an Abelian group acting diagonally on the product surface $Z$. All the known examples of infinite families can be recovered from our method and it can be generalized naturally to varieties of dimension $>2$. Finally we should point out the rich and unknown geometry that the following two theorems show.

Theorem (The case $|G| \leq 8$ ):

Let $S$ be a minimal desingularization of the quotient $Z / G$ where $Z$ is the product surface $F \times D$ and $G$ is an Abelian group acting faithfully on the curves $F, D$ and diagonally on $Z$; set $A=F / G$ and $B=D / G$. Assume that the canonical map of $S$ factorizes through $\pi_{2}: X \rightarrow B$. If $p_{g}(S)=m-1 \geq 3,|G| \leq 8$ and $g(F) \leq 3$ then only the following cases occur:

\begin{tabular}{|c|c|c|c|c|c|}
\hline$g(A)$ & $g(B)$ & $G$ & $g(F)$ & $g(D)$ & $K_{S}^{2}$ \\
\hline 0 & 0 & $\mathbb{Z} / 2 \times \mathbb{Z} / 2$ & 2 & $2 m-1$ & $4 m$ \\
\hline 0 & 0 & $\mathbb{Z} / 2 \times \mathbb{Z} / 2$ & 2 & $2 m-2$ & $4 m-6$ \\
\hline 0 & 0 & $\mathbb{Z} / 2 \times \mathbb{Z} / 2$ & 2 & $2 m-3$ & $4 m-8$ \\
\hline 0 & 1 & $\mathbb{Z} / 2 \times \mathbb{Z} / 2$ & 2 & $2 m-1$ & $4 m-4$ \\
\hline 1 & 0 & $\mathbb{Z} / 2$ & 2 & $m$ & $4 m-4$ \\
\hline 0 & 0 & $\mathbb{Z} / 2 \times \mathbb{Z} / 2$ & 3 & $2 m-1$ & $8 m$ \\
\hline 0 & 0 & $\mathbb{Z} / 2 \times \mathbb{Z} / 2$ & 3 & $2 m-2$ & $8 m-12$ \\
\hline 0 & 0 & $\mathbb{Z} / 2 \times \mathbb{Z} / 2$ & 3 & $2 m-3$ & $8 m-16$ \\
\hline 0 & 0 & $\mathbb{Z} / 2 \times \mathbb{Z} / 2 \times \mathbb{Z} / 2$ & 3 & $4 m+1$ & $8 m$ \\
\hline 0 & 0 & $\mathbb{Z} / 2 \times \mathbb{Z} / 2 \times \mathbb{Z} / 2$ & 3 & $4 m-1$ & $8 m-4$ \\
\hline 0 & 0 & $\mathbb{Z} / 2 \times \mathbb{Z} / 2 \times \mathbb{Z} / 2$ & 3 & $4 m-3$ & $8 m-8$ \\
\hline 0 & 0 & $\mathbb{Z} / 2 \times \mathbb{Z} / 2 \times \mathbb{Z} / 2$ & 3 & $4 m-5$ & $8 m-12$ \\
\hline 0 & 0 & $\mathbb{Z} / 2 \times \mathbb{Z} / 2 \times \mathbb{Z} / 2$ & 3 & $4 m-7$ & $8 m-16$ \\
\hline 0 & 1 & $\mathbb{Z} / 2 \times \mathbb{Z} / 2$ & 3 & $2 m-1$ & $8 m-8$ \\
\hline 0 & 1 & $\mathbb{Z} / 2 \times \mathbb{Z} / 2 \times \mathbb{Z} / 2$ & 3 & $4 m-3$ & $8 m-8$ \\
\hline 1 & 0 & $\mathbb{Z} / 2 \times \mathbb{Z} / 2$ & 3 & $2 m-1$ & $8 m-8$ \\
\hline 1 & 0 & $\mathbb{Z} / 2 \times \mathbb{Z} / 2$ & 3 & $2 m-2$ & $8 m-12$ \\
\hline 1 & 0 & $\mathbb{Z} / 2 \times \mathbb{Z} / 2$ & 3 & $2 m-3$ & $8 m-16$ \\
\hline 2 & 0 & & 3 & $m-1$ & $8 m-16$ \\
\hline
\end{tabular}

If $|G|>8$ only the case $G=\mathbb{Z} / 2 \times \mathbb{Z} / 8$ occurs, but it produces many different infinite families; see the proof of 4.6 and the proof of 4.7 for the last entry of the next table. More precisely: 
Theorem (The case $|G|>8$ ):

If $|G|>8$ then $G=\mathbb{Z} / 2 \times \mathbb{Z} / 8, g(F)=3, g(A)=0$. Moreover the actions are completely described and if $m \geq 4$ only the following invariants occur:

\begin{tabular}{|c|c|c|c|}
\hline$g(B)$ & $p_{g}$ & $g(D)$ & $K_{S}^{2}$ \\
\hline 0 & $m$ & $8 m-1$ & $8 m-6$ \\
\hline 0 & $m-1$ & $8 m-5$ & $8 m-16$ \\
\hline 0 & $m$ & $8 m-5$ & $8 m-10$ \\
\hline 0 & $m-1$ & $8 m-9$ & $8 m-20$ \\
\hline 0 & $m+1$ & $8 m+4$ & $8 m$ \\
\hline 0 & $m$ & $8 m$ & $8 m-4$ \\
\hline 0 & $m-1$ & $8 m-4$ & $8 m-8$ \\
\hline 0 & $m$ & $8 m$ & $8 m-3$ \\
\hline 0 & $m-1$ & $8 m-4$ & $8 m-7$ \\
\hline 0 & $m-2$ & $8 m-8$ & $8 m-11$ \\
\hline 0 & $m$ & $8 m+3$ & $8 m$ \\
\hline 0 & $m-1$ & $8 m-1$ & $8 m-4$ \\
\hline 0 & $m$ & $8 m-1$ & $8 m-4$ \\
\hline 0 & $m-1$ & $8 m-5$ & $8 m-8$ \\
\hline 0 & $m-1$ & $8 m-9$ & $8 m-12$ \\
\hline 0 & $m+1$ & $8 m+12$ & $8 m+7$ \\
\hline 0 & $m$ & $8 m+8$ & $8 m+3$ \\
\hline 0 & $m-1$ & $8 m+4$ & $8 m-1$ \\
\hline 1 & $m$ & $8 m+1$ & $8 m$ \\
\hline
\end{tabular}

Acknowledgment: I would like to thank F.Catanese for pointing out some evidence [5] which brought me to theorem 2.3, then M. Manetti for showing me a short way to exclude the genus- 5 case and the group of geometers of Dipartimento di Matematica del Politecnico di Torino, where I wrote the first version of this paper, for their supportive attitude.

\section{Notations.}

$S$ will be a surface with canonical map composed with the pencil $f: S \rightarrow B$ and also it will be the desingularization of $X=F \times D / G$ where $G$ is an Abelian group acting faithfully on the two curves $F, D$ with quotients $F / G=A$ and $D / G=B$ and acting diagonally on $F \times D$. In particular $f$ will factorize through the projection $X \rightarrow B$ and $F$ will be its fibre. Since we will be concerned with product surfaces we will denote by capital letters also the points of $A, B, D, B$ whenever it can arise no confusion. 


\section{The diagonal action}

An action of a group $G$ on a variety $Z$ is called faithful if no non-trivial element of $G$ acts trivially on $Z$. Let us consider an analytic faithful action of a finite group $G$ on $Z$. The isotropy subgroup (stabilizer) of a point $P \in Z$ is the subgroup $G_{P}$ of elements in $G$ fixing $P$. An action is free if $G_{P}=\{1\}$ for every $P \in Z$. Let $X=Z / G$ be the quotient variety with the natural projection $\pi_{G}: Z \rightarrow X$. A point $P \in Z$ is called a ramification point if $\pi_{G}$ is ramified at $P$ and $\pi_{G}(P)=[P]$ is called a branch point. It is well-known that all points in $\pi_{G}^{-1}[P]$ have the same multiplicity by $\pi_{G}$ and this number is called the branching order of $[P]$. If $G$ acts on two smooth curves $F, D$, we can define the diagonal action on $F \times D$ by $\left(x_{1}, x_{2}\right) \mapsto\left(\gamma x_{1}, \gamma x_{2}\right)$ for all $\left(\gamma, x_{1}, x_{2}\right) \in G \times F \times D$. We consider a finite group $G$ acting faithfully on two smooth curves $F, D$ and we denote $Z=F \times D, A=F / G, B=D / G$. Set $X=Z / G$ for the quotient by the diagonal action and denote by $\pi_{A}: X \rightarrow A, \pi_{B}: X \rightarrow B$ the two projections. In particular $\pi_{B}: X \rightarrow B$ is a constant moduli fibration meaning that all the smooth fibres are isomorphic to $F$. A similar description applies to $\pi_{A}: X \rightarrow A$ : its smooth fibres are isomorphic to $D$. Since the stabilizer of a point $P$ of a curve is a cyclic subgroup of $G$ then the finite map $\pi_{G}: Z \rightarrow X$ is branched at the isolated points $\left[\left(x_{1}, x_{2}\right)\right]$ such that $\left[\left(\gamma x_{1}, \gamma x_{2}\right)\right]=\left[\left(x_{1}, x_{2}\right)\right]$ for some $\gamma \in G \backslash\{\mathrm{id}\}$. These points are precisely the singular locus of $X$, in view of the purity of branch locus theorem. In particular $X$ has cyclic quotient singularities, hence Hirzebruch-Jung singularities only. Then if $\nu: S \rightarrow X$ is the minimal desingularization of $X$, [ [14] p. 64], the fibre of $\nu$ over each singular point of $X$ is an Hirzebruch-Jung string [cf.[2]]. Such desingularization is called a standard isotrivial surface after Serrano [14] and the induced fibrations $f_{1}: S \rightarrow A, f_{2}: S \rightarrow B$ are called standard isotrivial fibrations. In the rest of this paper $G$ will be an Abelian group acting faithfully on the smooth curves $F, D$ and we will also assume $g(F) \geq 2$ and $g(D) \geq 2$. Let $\Delta: G \times Z \rightarrow Z$ be the diagonal action with quotient $Z / G=X$ and with quotient map $\rho: Z \rightarrow X$. Since $G$ acts freely on $Z$ outside a finite set of points $\left\{P_{1}, \ldots P_{t}\right\} \subset Z$ with non-trivial stabilizer $G_{P_{i}}, X$ is a normal surface with only isolated rational singularities [[4] Satz 1.7]. Let $\operatorname{Sing}(X)=\left\{\rho\left(P_{1}\right), \ldots \rho\left(P_{t}\right)\right\} \subset Z$ be the singular locus of $X$ and let $j: X_{0} \rightarrow X$ be the natural inclusion. By well-known results on isolated quotient singularities cf.[12][Théorème pp. 169] we have $\mathcal{O}_{X}=j_{\star} \mathcal{O}_{X_{0}}=$ $\left(\rho_{\star} \mathcal{O}_{Z}\right)^{G}$ and $\omega_{X}=j_{\star} \omega_{X_{0}}=\left(\rho_{\star} \omega_{Z}\right)^{G}$. Moreover, since $G$ is Abelian, there is an action $\alpha: G \times X \rightarrow X$ induced by $\alpha^{\prime}: G \times Z \rightarrow Z$ defined by $\alpha^{\prime}:(g ;(x, y)) \mapsto(g x, y)$. We call $\alpha$ the quotient action. We set $Y=X / G$ and by a trivial computation we obtain $Y=A \times B$.

The next proposition is an extension of proposition 4.1 in [11] to a surface with isolated rational singularities.

Proposition 1.1 Let $\alpha: G \times X \rightarrow X$ be an action of an Abelian finite group $G$ on a surface $X$ with isolated rational singularities and let $\pi_{G}: X \rightarrow X / G$ be the projection. Set $X / G=Y$ and assume that $Y$ is a smooth surface. Then for all $\chi \in G^{\star}$, where $G^{\star}$ is the group of characters, there exists an invertible sheaf $L_{\chi}$ on $Y$ such that 


$$
\text { i) } \pi_{G_{\star}} \mathcal{O}_{X}=\oplus_{\chi \in G^{\star}} L_{\chi}^{-1}
$$

where $G$ acts on $L_{\chi}^{-1}$ via the character $\chi$ and the invariant summand $L_{i d}=\mathcal{O}_{Y}$. Furthermore we also have a decomposition into invertible sheaves of $\pi_{G_{\star}} \omega_{X}$ such that:

$$
\begin{array}{ll}
\text { ii) } & \left(\pi_{G_{\star}} \omega_{X}\right)^{\mathrm{inv}}=\omega_{Y} \\
\text { iii }) & \left(\pi_{G_{\star}} \omega_{X}\right)^{\chi}=\omega_{Y} \otimes L_{\chi^{-1}}
\end{array}
$$

where $G$ acts on $\left(\pi_{G_{\star}} \omega_{X}\right)^{\chi}$ via the character $\chi$.

Proof. i). Since $X$ is normal, $Y$ is smooth and $G$ is Abelian then by [1], $\pi_{G}$ is flat and the action $\alpha$ induces the desired splitting on $\pi_{G_{\star}} \mathcal{O}_{X}$.

ii). Let $\operatorname{Sing}(X)$ be the union of the singular points of $X, X_{0}=X \backslash \operatorname{Sing}(X)$, $Y_{0}=X_{0} / G$ and let $j: X_{0} \rightarrow X, i: Y_{0} \rightarrow Y, \pi_{G}^{0}: X_{0} \rightarrow Y_{0}$ be respectively the natural inclusions and the projection. Since $\pi_{G}^{0}$ is a smooth $G$-cover then by [11]] [prop 4.1] there exist $L_{\chi}^{0} \in \operatorname{Pic}\left(Y_{0}\right)$ such that $\pi_{G_{\star}}^{0} \mathcal{O}_{X_{0}}=\oplus_{\chi} \in G^{\star}\left(L_{\chi}^{0}\right)^{-1}$ and $\left(\pi_{G_{\star}}^{0} \omega_{X_{0}}\right)^{\chi}=\omega_{Y_{0}} \otimes L_{\chi^{-1}}^{0}$. Since $Y, Y_{0}$ are smooth and $Y \backslash Y_{0}$ is a finite set of points then by part i) we have $i^{\star} L_{\chi}=L_{\chi}^{0}$ and $i^{\star} \omega_{Y}=\omega_{Y_{0}}$ then $i_{\star}\left(\omega_{Y^{0}} \otimes L_{\chi^{-1}}^{0}\right)=\omega_{Y} \otimes L_{\chi^{-1}}$. We want to show that:

$$
\left(\pi_{G_{\star}} \omega_{X}\right)^{\chi}=\omega_{Y} \otimes L_{\chi^{-1}} .
$$

Since $\omega_{X}=j_{\star} \omega_{X_{0}}$ then $i_{\star} \pi_{G_{\star}}^{0} \omega_{X_{0}}=\pi_{G_{\star}} \omega_{X}$. On the other hand $i_{\star}\left(\left(\pi_{G_{\star}}^{0} \omega_{X_{0}}\right)^{\chi}\right)$ and $\left(i_{\star} \pi_{G_{\star}}^{0} \omega_{X_{0}}\right)^{\chi}$ coincide outside a finite set of points and $i_{\star}\left(\left(\pi_{G_{\star}}^{0} \omega_{X_{0}}\right)^{\chi}\right)=\left(i_{\star} \pi_{G_{\star}}^{0} \omega_{X_{0}}\right)^{\chi}$ since $Y$ is smooth (normal). Then $\left(\pi_{G_{\star}} \omega_{X}\right)^{\chi}=i_{\star}\left(\left(\omega_{Y^{0}} \otimes L_{\chi^{-1}}^{0}\right)=\omega_{Y} \otimes L_{\chi^{-1}} . \quad \dashv\right.$

If $G$ is an Abelian finite group acting faithfully on two smooth curves $F$ and $D$, the diagonal $G$-action on the product surface $Z=F \times D$ and the quotient action on the surface $X=Z / G$ forces some structure on the invariants of $X$ which can be read from the $G$-action on $F$ and $D$. Then we are led naturally to the following definition:

Definition 1.2 Let $G$ be an Abelian group acting faithfully on two smooth curves, $F$ and $D$ where $g(F) \geq 2, g(D) \geq 2$. Let $Z=F \times D$ and let $\Delta: G \times Z \rightarrow Z$ be the diagonal action with quotient $Z / G=X$. We will say that $X$ is a $G$ - sandwich surface with top $F \times D$ and base $A \times B$, where $A=F / G, B=D / G$.

The next lemma relates the invariants of a $G$ - sandwich surface $X$ with the invariants of the curves which produce $X$.

Lemma 1.3 If $X$ is a $G$ - sandwich surface with top $Z=F \times D$ and base $A \times B$ then

$$
\begin{aligned}
& H^{0}\left(X, \Omega_{X}^{1}\right)=H^{0}\left(A, \Omega_{A}^{1}\right) \oplus H^{0}\left(B, \Omega_{B}^{1}\right) \\
& H^{0}\left(X, \omega_{X}\right)=H^{0}\left(Z, \omega_{Z}\right)^{G}
\end{aligned}
$$


Proof. Let $\nu: W \rightarrow X$ be a minimal resolution of $X$. By [8][satz 1, p.99] we have

$$
\begin{aligned}
& H^{0}\left(W, \Omega_{W}^{1}\right)=H^{0}\left(A, \Omega_{A}^{1}\right) \oplus H^{0}\left(B, \Omega_{B}^{1}\right) ; \\
& H^{0}\left(W, \omega_{W}\right)=H^{0}\left(Z, \omega_{Z}\right)^{G} .
\end{aligned}
$$

Since $X$ has rational singularities then $\nu_{\star} \Omega_{W}^{1}=i_{\star} \Omega_{X_{0}}^{1}={ }_{\text {def }} \Omega_{X}^{1}$ and $\nu_{\star} \omega_{W}=\omega_{X}$. The claim is now obvious. $\dashv$

If $X$ is a $G$-sandwich surface with top $Z=F \times D$ and $H^{0}\left(F, \Omega_{F}^{1}\right)=\oplus_{\chi \in G^{\star}} V_{1, \chi}$, $H^{0}\left(D, \Omega_{D}^{1}\right)=\oplus_{\chi \in G^{\star}} V_{2, \chi}$ are the decompositions in subspaces where $G$ acts on $V_{i, \chi}$ via the character $\chi$, there is a nice decomposition on the vector space $H^{0}\left(X, \omega_{X}\right)$ which is a sort of Künneth formula for an Abelian quotient of a direct product.

Theorem 1.4 Let $X=Z / G$ be a G-sandwich surface with top $Z=F \times D$ and base $Y=A \times B$ and let $H^{0}\left(F, \Omega_{F}^{1}\right)=\oplus_{\chi \in G^{\star}} V_{1, \chi}, H^{0}\left(D, \Omega_{D}^{1}\right)=\oplus_{\chi \in G^{\star}} V_{2, \chi}$ be the decompositions in subspaces where $G$ acts on $V_{i, \chi}$ via the character $\chi$. Then

$$
H^{0}\left(X, \omega_{X}\right)=\oplus_{\chi \in G^{\star}} V_{1, \chi} \otimes V_{2, \chi^{-1}}
$$

Proof. By $1.3 H^{0}\left(X, \omega_{X}\right)=H^{0}\left(Z, \Omega_{Z}^{2}\right)^{G}$. On the other hand by the Künneth formula $H^{0}\left(Z, \Omega_{Z}^{2}\right)=\left(H^{0}\left(A, \Omega_{A}^{1}\right) \otimes H^{0}\left(B, \Omega_{B}^{1}\right)\right)$, then $H^{0}\left(Z, \Omega_{Z}^{2}\right)^{G}=\left(H^{0}\left(A, \Omega_{A}^{1}\right) \otimes\right.$ $\left.H^{0}\left(B, \Omega_{B}^{1}\right)\right)^{G}$, that is $H^{0}\left(X, \omega_{X}\right)=\left(\oplus_{\chi, \chi^{\prime} \in G^{\star}} V_{1 \chi} \otimes V_{2 \chi^{\prime}}\right)^{G}=\oplus_{\chi \in G^{\star}} V_{1 \chi} \otimes V_{2 \chi^{-1}} \quad \dashv$

We have looked at the roof of $\mathrm{X}$. Now we pay attention to the floor and we discover that the pieces of the decomposition $H^{0}\left(X, \omega_{X}\right)=\oplus_{\chi \in G^{\star}} V_{1, \chi} \otimes V_{2, \chi^{-1}}$ induced by the diagonal action can be related to the pieces on $Y$ produced by the quotient action $\alpha$.

Proposition 1.5 With the same hypotheses as for Theorem 1.4, let $\pi_{G}: X \rightarrow Y$ be the projection obtained by the quotient action $\alpha$ with associated splitting: $\pi_{G_{\star}} \mathcal{O}_{X}=$ $\oplus_{\chi \in G^{\star}} L_{\chi}^{-1}$. Then for all $\chi \in G^{\star}$ we have

$$
H^{0}\left(X, \omega_{X}\right)^{\chi} \simeq H^{0}\left(Y, \omega_{Y} \otimes L_{\chi^{-1}}\right) \simeq V_{1, \chi} \otimes V_{2, \chi^{-1}}
$$

Proof. By 1.1 $\left(\pi_{G_{\star}} \omega_{X}\right)^{\chi}=\omega_{Y} \otimes L_{\chi^{-1}}$. Then $H^{0}\left(X, \omega_{X}\right)^{\chi} \simeq H^{0}\left(Y, \omega_{Y} \otimes L_{\chi^{-1}}\right)$. On the other hand the action $\alpha$ is induced by the action $\alpha^{\prime}: G \times Z \rightarrow Z$ which operates trivially on the second factor. Then by 1.4 we have $H^{0}\left(X, \omega_{X}\right)^{\chi}=V_{1, \chi} \otimes V_{2, \chi^{-1}}$; that is $H^{0}\left(Y, \omega_{Y} \otimes L_{\chi^{-1}}\right)=V_{1, \chi} \otimes V_{2, \chi^{-1}} . \quad \dashv$

\section{Surfaces with canonical map composed with a pencil}

Surfaces do not usually come equipped with a fibration, but when they do and if the fibration is the map associated with the canonical linear system, the interplay between 
the genus and other additional structure on the fibres, the genus of the base curve and the invariants naturally attached to the surface provide a great deal of information.

A surjective morphism with connected fibre $F, f: S \rightarrow B$ of a smooth projective surface $S$ onto a genus $b$ smooth curve $B$ is called a genus $b$ pencil of curves of genus $g$ if $g$ is the arithmetic genus of $F$. From now on we assume that $S$ is a surface of general type with $p_{g} \geq 2$. We say that the canonical linear system $\left|K_{S}\right|$ is composed with a pencil if the canonical image $\Sigma=\Phi_{\left|K_{S}\right|}(S)$ is a curve; in this case let $\epsilon: S^{\prime} \rightarrow S$ be the elimination of the base points of the moving part of $\left|K_{S}\right|$, then, taking the Stein factorization, we get a genus- $b$ pencil $f: S^{\prime} \rightarrow B$ and a morphism $B \rightarrow \Sigma$. We call the induced fibration $f: S^{\prime} \rightarrow B$ the canonical fibration. Since we will deal with singular surfaces we will recall Beauville's definition of canonical map for a singular surface of general type, [3] [pp.127]:

Definition 2.1 Let $X$ be a singular surface. Let $\nu: S \rightarrow X$ be a birational morphism where $S$ is a smooth surface. The rational map $\Phi_{\left|K_{X}\right|}={ }_{\operatorname{def}} \Phi_{\left|K_{S}\right|} \circ(\nu)^{-1}$ is called the canonical map of $X$. Moreover we put $p_{g}(X)=p_{g}(S)$ and one says that $X$ is of general type if $S$ is of general type.

Let us assume that the surface $X$ is of general type; by the unicity of the minimal model, the definition of $\Phi_{\left|K_{X}\right|}$ does not depend on the choice of $\nu$. In particular $\Phi_{\left|K_{X}\right|}(X)$ is a curve if and only if $\Phi_{\left|K_{S}\right|}(S)$ is a curve. Now we define a suitable class of surfaces with canonical map composed with a pencil. This class includes all the known examples of surfaces with isotrivial canonical fibration; see: [3], [16], [17], [9], [20].

Definition 2.2 We shall say that $S$ is a $G$-sandwich canonically $g$-fibred surface on $B$ with top $F \times D$ and base $A \times B$ (G-canonical for short) if 1$) S$ is a smooth model of a $G$ - sandwich surface $X$ with top $F \times D$ and base $A \times B$ [see 1.9]; 2) $\Phi_{\left|K_{S}\right|}(S)=B$, $g=g(F)$; 3) the induced fibration $f: S \rightarrow B$ factorizes through $\pi_{B}: X \rightarrow B$.

In the following theorem we will prove an effective method to characterize $G$-sandwich canonically $g$-fibred surfaces. This theorem relies on theorem 1.4.

Theorem 2.3 Let $X$ be a $G$-sandwich surface with top $Z=F \times D$ and base $Y=A \times B$ with $p_{g} \geq 2$ and let $\nu: S \rightarrow X$ be a minimal desingularization of $X$. Let $H^{0}\left(F, \Omega_{F}^{1}\right)=$ $\oplus_{\chi \in G^{\star}} V_{1, \chi}, H^{0}\left(D, \Omega_{D}^{1}\right)=\oplus_{\chi \in G^{\star}} V_{2, \chi}$ be the decompositions into subspaces where $G$ acts on $V_{i, \chi}, i=1,2$, via the character $\chi$. Then $S$ is a G-sandwich canonically $g$-fibred surface on $B$ if and only if there exists an unique $\chi_{0} \in G^{\star}$ such that the following two conditions hold:

$$
\begin{aligned}
& \text { i) } V_{1, \chi_{0}} \otimes V_{2, \chi_{0}^{-1}} \neq 0 \\
& \text { ii) } \operatorname{dim}_{\mathbb{C}} V_{1, \chi_{0}}=1, \operatorname{dim}_{\mathbb{C}} V_{2, \chi_{0}^{-1}}=p_{g} .
\end{aligned}
$$

Proof. If there exists an unique $\chi_{0} \in G^{\star}$ such that $\operatorname{dim}_{\mathbb{C}} V_{1, \chi_{0}} \neq 0, \operatorname{dim}_{\mathbb{C}} V_{2, \chi_{0}^{-1}} \neq 0$ then by 1.1 and $1.5 H^{0}\left(x, \omega_{X}\right)^{\chi}=0$ for all $\chi \neq \chi_{0}$. In particular by $1.5 H^{0}\left(X, \omega_{X}\right)=$ 
$V_{1, \chi_{0}} \otimes V_{2, \chi_{0}^{-1}}$. Now, if $\operatorname{dim}_{\mathbb{C}} V_{1, \chi_{0}}=1$ and $\phi, \psi \in H^{0}\left(X, \omega_{X}\right)$, then $\psi / \phi=\mu(y)$ is a meromorphic function which depends only on $y \in B$; whence $\Phi_{\left|K_{X}\right|}$ factors through $f_{2}: X \rightarrow B$. Conversely, if $\Phi_{\left|K_{X}\right|}$ is composed with the pencil $f_{2}: X \rightarrow B$ and there exist $\chi, \chi^{\prime} \in G^{\star}$ such that $V_{1, \chi} \otimes V_{2, \chi^{-1}} \neq 0$ and $V_{1, \chi^{\prime}} \otimes V_{2, \chi^{\prime-1}} \neq 0$ or there exists $\chi_{0} \in G^{\star}$ such that $\left(\operatorname{dim}_{\mathbb{C}} V_{1, \chi_{0}}\right) \times\left(\operatorname{dim}_{\mathbb{C}} V_{2, \chi_{0}^{-1}}\right) \geq 2\left(\operatorname{dim}_{\mathbb{C}} V_{2, \chi_{0}^{-1}}\right)$ then there exist $\eta_{1}, \eta_{2} \in H^{0}\left(F, \Omega_{F}^{1}\right)$ and $\sigma_{1}, \sigma_{2} \in H^{0}\left(D, \Omega_{D}^{1}\right)$ such that $\omega_{i}=\eta_{i} \wedge \sigma_{i}, i=1,2$ are 2-forms on $X$ and they induce a moving linear system on the fibres $F$ of $f_{2}: X \rightarrow B$. In particular $\Phi_{\left|K_{X}\right|}(F)$ can not be a point; a contradiction. The same argument shows that $\operatorname{dim}_{\mathbb{C}} V_{1, \chi_{0}}=1$ and $\operatorname{dim}_{\mathbb{C}} V_{2, \chi_{0}^{-1}}=p_{g} . \quad \dashv$

Surfaces with canonical map composed with a pencil are very exceptional in the theory of surfaces of general type. In particular there are strong bounds on some of their invariants. The next proposition is a specialization of these bounds to the case of a $G$-sandwich canonically $g$-fibred surface.

Theorem 2.4 If $S$ is a $G$-sandwich canonically g-fibred surface on $B$ with top $F \times D$ and base $A \times B$ with $p_{g}(X) \geq 11$ and $g=g(F)$ then $2 \leq g \leq 5$ and only the following cases can occur:

$$
\text { A) } g(A)=q(X) \leq 2 \text { and } g(B)=0 \text {; }
$$

or

$$
\text { B) } g(A)=0 \text { and } g(B)=q(X)=1 .
$$

Moreover if $p_{g} \geq \max \{85-2 q(X), 44\}$ then $g=5$ does not occur.

Proof. By 2.1 there is a birational morphism $\eta: S \rightarrow X$ where $\Phi_{\left|K_{S}\right|}$ is composed with the pencil $f=f_{2} \circ \eta$. It is easy to see that the moving part of $\left|K_{S}\right|$ is without base points. In particular $F$ is the generic fibre $F$ of $f$ and then the bound $2 \leq g \leq 5$ on $g(F)$ is in [3] [Proposition 2.1]. Since $q(X)=g(A)+g(B)$ then $A$ ) and $B$ ) are easily obtained by [17][proposition]. Now we show that $g=5$ and $p_{g} \geq 44$ does not occur. In fact if $g=5$ then by [15] (see also [20]) $K_{S}=8 H+V+f^{\star}(\gamma)$ where $p_{g}(S)=h^{0}\left(B, \mathcal{O}_{B}(\gamma)\right.$ ), $V F=0, H F=1$. In particular if $g(B)=0, K_{S} H \geq 3$ since $p_{g} \geq 44$ and $K_{S} H \geq 1$ if $g(B)=1$. Then $K_{S}^{2} \geq 8\left(p_{g}(S)+2\right)$ if $g(B)=0$ and $K_{S}^{2} \geq 8\left(p_{g}(S)+1\right)$ if $g(B)=1$. On the other hand by [14][Proposition 5.3] the Euler characteristic satisfies: $2 c_{2}(S) \geq K_{S}^{2}$, i.e. $c_{2}(S) \geq 4\left(p_{g}(S)+2\right)$ in case $\left.A\right)$ and $c_{2}(S) \geq 4\left(p_{g}(S)+1\right)$ in case $\left.B\right)$ : a contradiction with the Noether formula. $\dashv$

¿From Theorem 2.4 we see that to produce a $G$-sandwich canonically $g$-fibred surface with large invariants we must try to construct surfaces with $p_{g}(S) \gg 0$. On the other hand the above restriction on the genus of $F, A, B$ enable us to build all the $G$-sandwich canonically $g$-fibred surfaces. In fact the two conditions in theorem 2.3 show a procedure to manifest all these surfaces. The following remark will be useful: 
Remark 2.5 If $S$ is a $G$-sandwich canonically $g$-fibred surface on $B$ then there exist $\eta \in H^{0}\left(F, \Omega_{F}^{1}\right), \omega_{1}, \ldots, \omega_{p_{g}(S)} \in H^{0}\left(D, \Omega_{D}^{1}\right)$ such that

$$
\left\langle\eta \wedge \omega_{1}, \ldots, \eta \wedge \omega_{p_{g}(S)}\right\rangle
$$

gives a basis of $H^{0}\left(S, \omega_{S}\right)$. Moreover $\operatorname{div}(\eta) \in \operatorname{Div}(F)$ is a $G$-invariant divisor on $F$.

Proof. The proof is trivial if in 2.3 we put $V_{1, \chi_{0}}=\eta \mathbb{C}$ and $V_{2, \chi_{0}^{-1}}=\oplus_{i=1}^{p_{g}(S)} \omega_{i} \mathbb{C}$.

We will be concerned mostly with $G$-canonical surfaces where $G$ is a product of some copies of $\mathbb{Z} / 2$. In-fact we will use tacitly the following description of quotient surfaces $X=Z / G$ where all the stabilizers are isomorphic to $\mathbb{Z} / 2$.

Theorem 2.6 Let $Z$ be a nonsingular surface with $G$ acting freely outside the finite set of points $\left\{P_{1}, \ldots P_{t}\right\} \subset Z$ with stabilizer $G_{P_{i}}=\mathbb{Z} / 2$ for $i=1, \ldots, t$. Let $\pi: Z \rightarrow$ $X=Z / G$ be the quotient map and let $\nu: S \rightarrow X$ be the minimal resolution (i.e. the blow up of the $t$ nodes of $X)$. Then if $n=|G|$ we have:

$$
\begin{array}{cl}
\text { i) } & K_{S}^{2}=K_{X}^{2}=\frac{1}{n} K_{Z}^{2} \\
\text { ii) } & \chi\left(\mathcal{O}_{S}\right)=\chi\left(\mathcal{O}_{X}\right)=\frac{1}{n}\left(\chi\left(\mathcal{O}_{Z}\right)+\frac{t}{4}\right) \\
\text { iii) } & \text { If } Z \text { is a minimal surface of general type then so is } S .
\end{array}
$$

Proof. See cf. [1][p. 295]. $\dashv$

Finally we recall that the aim of this paper is to look into infinite families of surfaces with isotrivial canonical map. By [3][Proposition 1.7] the minimal surfaces $S$ of general type with $\chi\left(\mathcal{O}_{S}\right)<N$ (or $K_{S}^{2}<N$ or $p_{g}<N$ ) are in a limited family. Then, given $m \in \mathbb{Z}^{+}, m \gg 0$ we want surfaces with $p_{g}=m$ and isotrivial canonical fibration. By 2.4 the irregularity $q=q(S)=g(A)+g(B)$ is at most 2 and if $p_{g} \gg 0$, $2 \leq g=g(F) \leq 4$. All known examples are with $g=2$ or $g=3$. We postpone the case $g=4$ and the exceptional limited families with $g \geq 5$ to a forthcoming paper. We will classify all the $G$-sandwich canonically $g$-fibred surfaces with $p_{g} \gg 0$ and $g=2$ or $g=3$. The seven cases to classify are listed below (see 2.4):

\begin{tabular}{|c|c|c|c|}
\hline Case & $g(F)$ & $g(A)$ & $g(B)$ \\
\hline$B^{(2)}$ & 2 & 0 & 1 \\
\hline$A_{0}^{(2)}$ & 2 & 0 & 0 \\
\hline$A_{1}^{(2)}$ & 2 & 1 & 0 \\
\hline$B^{(3)}$ & 3 & 0 & 1 \\
\hline$A_{0}^{(3)}$ & 3 & 0 & 0 \\
\hline$A_{1}^{(3)}$ & 3 & 1 & 0 \\
\hline$A_{2}^{(3)}$ & 3 & 2 & 0 \\
\hline
\end{tabular}




\section{$3 \quad g(F)=2$}

The outline of the classification proof of infinite families of $G$-sandwich canonically 2 -fibred surfaces is the same as that of genus- 3 case. On the other hand it is easier and it is clear how it works. We describe all the genus- 2 infinite families even in the known cases. Moreover we will prove a unicity theorem which was a gap in this theory. We will need the following well-known result:

Lemma 3.1 If $G$ is a finite Abelian group acting on a smooth curve $C$ then $C \rightarrow C / G$ has at least two branch points .

Proof. See cf. [14][Lemma 5.7]. $\quad-$

Theorem 2.3 works if we have classified all the $G$-actions on a curve $F$ of genus 2 with an invariant canonical divisor: see remark 2.5. In particular the splitting $H^{0}\left(F, \Omega_{F}^{1}\right)=\oplus_{\chi \in G^{\star}} V_{1, \chi}$ has to be computed:

Lemma 3.2 Let $G$ be an Abelian group acting on a smooth genus-2 curve $F$ with quotient $F / G=A$. Let $\eta \in H^{0}\left(F, \Omega_{F}^{1}\right)$ be a 1-form such that $\operatorname{div}(\eta)$ is a $G$-invariant divisor and let $H^{0}\left(F, \Omega_{F}^{1}\right)=\oplus_{\chi \in G^{\star}} V_{1, \chi}$ be the decompositions into subspaces where $G$ acts on $V_{1, \chi}$ via the character $\chi$. Then only the following cases occur:

\begin{tabular}{|l|c|l|}
\hline$g(A)$ & $G$ & $H^{0}\left(F, \Omega_{F}^{1}\right)$ \\
\hline 1 & $\mathbb{Z} / 2$ & $V_{1, \mathrm{id}} \oplus V_{1, \chi}$ \\
\hline 0 & $\mathbb{Z} / 2$ & $V_{1, \chi}$ \\
\hline 0 & $\mathbb{Z} / 3$ & $V_{1, \chi} \oplus V_{1, \chi^{2}}$ \\
\hline 0 & $\mathbb{Z} / 4$ & $V_{1, \chi} \oplus V_{1, \chi^{3}}$ \\
\hline 0 & $\mathbb{Z} / 5$ & $V_{1, \chi} \oplus V_{1, \chi^{3}}$ \\
\hline 0 & $\mathbb{Z} / 6$ & $V_{1, \chi} \oplus V_{1, \chi^{5}}$ \\
\hline 0 & $\mathbb{Z} / 2 \times \mathbb{Z} / 2$ & $V_{1, \chi_{1}} \oplus V_{1, \chi_{2}}$ \\
\hline
\end{tabular}

where $\chi$ is a generator of $(\mathbb{Z} / d)^{\star}$ if $d=2,3,4,5,6$ and $\chi_{1}, \chi_{2}$ generate $(\mathbb{Z} / 2 \times \mathbb{Z} / 2)^{\star}$.

Proof. The claim follows from 3.1 and from the well-known argument by Hurwitz to compute all the possible ramification indexes. -1

Our aim is to classify all $G$-sandwich canonically 2-fibred surfaces with $p_{g} \gg 0$. We start with the case $B^{2}$ in table (3).

Proposition 3.3 If $S$ is a $G$-sandwich canonically 2-fibred surface on an elliptic curve with $p_{g}(S)=m \geq 2$ then $S$ is the minimal desingularization of $X=Z / G$ where $G=\mathbb{Z} / 2 \times \mathbb{Z} / 2$ and it acts diagonally on $Z=F \times D, g(F)=2, g(D)=2 m+1$, $g(A)=0, g(B)=1$. Moreover $X$ has $8 m$ nodes, $\Phi_{\left|K_{S}\right|}$ is composed with the pencil $S \rightarrow B$ and $K_{S}^{2}=4 \chi\left(\mathcal{O}_{S}\right)$. 
Proof. Let $S$ be a $G$-canonical surface with $\Phi_{\left|K_{S}\right|}$ composed with the pencil $f_{2}: S \rightarrow B$ where $g(B)=1$ and $g(F)=2$. By 2.5 there is a $G$-invariant 1 -form on $F$. By 3.2 all these $G$ actions are known. Then we have to study all the $G$-covers $D \rightarrow B$ such that the decomposition families $\left\{V_{1, \chi}\right\}_{\chi \in G^{\star}},\left\{V_{2, \chi}\right\}_{\chi \in G^{\star}}$ satisfy the condition of 2.3. In particular we want the character $\chi_{0}$. Since $g(B)=1$ then by 2.4, $g(A)=0$. Now we follow the list in 3.2 table (4).

The case $\boldsymbol{G}=\mathbb{Z} / \mathbf{2}$ is impossible. In fact $H^{0}\left(F, \Omega_{F}^{1}\right)=V_{1, \chi_{0}}$, then $\operatorname{dim}_{\mathbb{C}} V_{1, \chi_{0}}=2$ contradicting 2.3 .

The cases $\boldsymbol{G}=\mathbb{Z} / \boldsymbol{d}$ where $d=3,4,5,6$ are impossible. The proofs are the same. We explain the proof of $d=3$ only. The Galois map $D \rightarrow B$ is given by $\mathcal{L}_{\chi}, \mathcal{L}_{\chi^{2}} \in \operatorname{Pic}(B)$ and two effective divisors $D_{1}, D_{2}$ such that:

$$
\left\{\begin{aligned}
3 \mathcal{L}_{\chi} & =D_{1}+2 D_{2} \\
\mathcal{L}_{\chi^{2}} & =2 \mathcal{L}_{\chi}-D_{2}
\end{aligned}\right.
$$

By 2.3 we have $V_{2, \chi}=0$ or $V_{2, \chi^{2}}=0$. Since $V_{2, \chi^{2}}=H^{0}\left(D, \Omega_{D}^{1}\right)^{\chi^{2}}=H^{0}\left(B, \mathcal{O}_{B}\left(\mathcal{L}_{\chi}\right)\right) \neq 0$ then $V_{2, \chi}=0$. Since $V_{2, \chi}=H^{0}\left(B, \mathcal{O}_{B}\left(\mathcal{L}_{\chi^{2}}\right)\right), \operatorname{deg}\left(\mathcal{L}_{\chi^{2}}\right)=0$. We $\operatorname{set} \operatorname{deg}\left(\mathcal{L}_{\chi}\right)=l_{\chi}$, $\operatorname{deg}\left(D_{1}\right)=d_{1}, \operatorname{deg}\left(D_{2}\right)=d_{2}$. Taking the degrees, we have $3 l_{\chi}=d_{1}+2 d_{2}$ and $2 l_{\chi}=d_{2}$, that is $l_{\chi}=d_{1}=d_{2}=0$. In particular $p_{g}(S)=0$.

$\boldsymbol{G}=\mathbb{Z} / \mathbf{2} \times \mathbb{Z} / \mathbf{2}$. In this case the covering $D \rightarrow B$ is given by the data $\mathcal{L}_{\chi_{1}}, \mathcal{L}_{\chi_{2}}, \mathcal{L}_{\chi_{3}} \in \operatorname{Pic}(B)$ where $\left\{1, \chi_{1}, \chi_{2}, \chi_{3}\right\}=G^{\star}$ and three effective divisors on $B$, $D_{1}, D_{2}, D_{3}$ such that:

$$
\left\{\begin{aligned}
2 \mathcal{L}_{\chi_{1}} & =D_{1}+D_{3} \\
2 \mathcal{L}_{\chi_{2}} & =D_{2}+D_{3} \\
\mathcal{L}_{\chi_{3}} & =\mathcal{L}_{\chi_{1}}+\mathcal{L}_{\chi_{2}}-D_{3}
\end{aligned}\right.
$$

By 2.3 and 3.2, $V_{2, \chi_{1}}=0$ or $V_{2, \chi_{2}}=0$. Assume $0=V_{2, \chi_{1}}=H^{0}\left(B, \mathcal{O}_{B}\left(\mathcal{L}_{\chi_{1}}\right)\right.$. In particular $\operatorname{deg}\left(\mathcal{L}_{\chi_{1}}\right)=0$. Then, taking the degrees, we obtain $l_{\chi_{1}}=d_{1}=d_{3}=0$, $2 l_{\chi_{2}}=d_{2}, l_{\chi_{2}}=l_{\chi_{3}}$. Since $D_{i}$ is effective we have $D_{1}=D_{3}=0$ and $\mathcal{L}_{\chi_{1}}$ is a 2 -torsion non-trivial divisor since $D$ is connected. Now if we set $l_{\chi_{2}}=m$ we have the claim. $\dashv$

Remark 3.4 This unique family is described by Beauville in [3][Example 2.6 p. 127].

Now we describe the case $A_{1}^{2}$ in the table (3).

Proposition 3.5 If $S$ is a $G$-sandwich canonically 2-fibred surface on a rational curve with $q(S)=1, p_{g}(S)=m \geq 2$ then $S$ is the minimal desingularization of $X=Z / G$ where $G=\mathbb{Z} / 2$ acts diagonally on $Z=F \times D, g(F)=2, g(D)=m+1, g(A)=1$, $g(B)=0$. Moreover $X$ has $4 m$ nodes, $\Phi_{\left|K_{S}\right|}$ is composed with $S \rightarrow B$ and $K_{S}^{2}=$ $4 \chi\left(\mathcal{O}_{S}\right)$. 
Proof. We suppose that $\left|K_{X}\right|$ is composed with $f_{2}: X \rightarrow B$ where $g(B)=0$. Since $1=q(X)=h^{0}\left(Z, \Omega_{Z}^{1}\right)^{G}$ then $g(A)=1$. Furthermore, by definition, $g(F)=2$ and therefore by 3.2, $G=\mathbb{Z} / 2$ and $H^{0}\left(F, \Omega_{F}^{1}\right)=H^{0}\left(F, \Omega_{F}^{1}\right)^{\text {id }} \oplus H^{0}\left(F, \Omega_{F}^{1}\right)^{\chi}$ where $G^{\star}=\{1, \chi\}$. From 2.3 it follows that each hyperelliptic covering $D \rightarrow B$ branched on $2 m+4$ points gives a solution.

Remark 3.6 This infinite family is described also by Beauville in [9]/Variante p. 126].

We finish this section with the case $A_{0}^{2}$ in the table (3). As far as we know there are examples for the case $A_{0}^{2}$ only in [16].

Proposition 3.7 If $S$ is a $G$-sandwich canonically 2-fibred surface on a rational curve, with $q(S)=0, p_{g}(S)=m-1 \geq 2$ then $S$ is the minimal desingularization of $X=Z / G$ where $G=\mathbb{Z} / 2 \times \mathbb{Z} / 2$ acts diagonally on $Z=F \times D, g(F)=2, X$ has $t$ nodes and $S$ is in one of the following three classes:

$$
\begin{array}{rlll}
I) & g(D)=2 m-1 & K_{S}^{2}=4 \chi\left(\mathcal{O}_{S}\right) & t=8 m+8 \\
I I) & g(D)=2 m-2 & K_{S}^{2}=4 \chi\left(\mathcal{O}_{S}\right)-6 & t=8 m+12 \\
I I I) & g(D)=2 m-3 & K_{S}^{2}=4 \chi\left(\mathcal{O}_{S}\right)-8 & t=8 m+16
\end{array}
$$

Proof. We can exclude the cases $G=\mathbb{Z} / d, d=2,3,4,5,6$ of 3.2 as in 3.3. Let $G=\mathbb{Z} / 2 \times \mathbb{Z} / 2$. Since $B=\mathbb{P}^{1}$ then $\operatorname{Pic}\left(\mathbb{P}^{1}\right)=\mathbb{Z}$ and to obtain the solutions we need to solve the degree system associated to (6) with the condition $H^{0}\left(B, \omega_{B}\left(\mathcal{L}_{\chi_{1}}\right)\right)=0$. Since $H^{0}\left(B, \omega_{B}\left(\mathcal{L}_{\chi_{1}}\right)\right) \simeq H^{0}\left(\mathbb{P}^{1}, \mathcal{O}_{\mathbb{P}^{1}}\left(l_{\chi_{1}}-2\right)\right)=0$ and $\operatorname{deg}\left(\mathcal{L}_{\chi_{1}}\right)>0$ the constraint is $\operatorname{deg}\left(\mathcal{L}_{\chi_{1}}\right)=1$. Taking the degrees in (6) we obtain:

$$
\begin{aligned}
\text { I) } & d_{1}=2, d_{2}=2 m, d_{3}=0, l_{\chi_{1}}=1, l_{\chi_{2}}=m, l_{\chi_{3}}=m+1 \\
I I) & d_{1}=1, d_{2}=2 m-1, d_{3}=1, l_{\chi_{1}}=1, l_{\chi_{2}}=m l_{\chi_{3}}=m \\
I I I) & d_{1}=0, d_{2}=2 m-2, d_{3}=2, l_{\chi_{1}}=1, l_{\chi_{2}}=m, l_{\chi_{3}}=m-1,
\end{aligned}
$$

which are the desired solutions. -1

We have shown the cases with $g(F)=2$ in the table (11).

\subsection{The geometrical construction of the three families of 3.7}

We want to construct the surfaces of 3.7 in a more geometrical way. We consider five distinct points $Q_{1}, Q_{2}, Q_{3}, Q_{4}, Q_{5} \in \mathbb{P}^{1}=A$. Let $\rho_{E}: E \rightarrow A$ and $\rho_{E^{\prime}}: E^{\prime} \rightarrow A$ be the $\mathbb{Z} / 2$ - covers branched on $Q_{1}, Q_{2}, Q_{3}, Q_{4}$ and on $Q_{2}, Q_{3}, Q_{4}, Q_{5}$ respectively. Set $\rho_{E}^{-1}\left(Q_{i}\right)=Q_{i}^{E}, i=1,2,3,4, \rho_{E}^{-1}\left(Q_{5}\right)=P_{1}^{E}+P_{2}^{E}$ and set $\rho_{E^{\prime}}^{-1}\left(Q_{i}\right)=Q_{i}^{E^{\prime}}, i=2,3,4,5$, $\rho_{E^{\prime}}^{-1}\left(Q_{1}\right)=P_{1}^{E^{\prime}}+P_{2}^{E^{\prime}}$. Moreover let $i_{E}: E \rightarrow E, i_{E^{\prime}}: E^{\prime} \rightarrow E^{\prime}$ be the involutions associated to $\rho_{E}, \rho_{E^{\prime}}$ respectively. Let $C$ be the normalization of the fibre product $E \times{ }_{A} E^{\prime}$, thus $g(C)=2$ and the natural map $\rho_{1}: C \rightarrow A$ is a $G=\mathbb{Z} / 2 \times \mathbb{Z} / 2$ cover. We call $\rho_{1}^{-1}\left(Q_{i}\right)=Q_{i 1}^{C}+Q_{i 2}^{C}, i=1,2,3,4,5$; in particular $\rho_{1}^{\star}\left(Q_{i}\right)=2 Q_{i 1}^{C}+2 Q_{i 2}^{C}$ 
where $i=1,2,3,4,5$. By abuse of notation we put $G=\left\langle i_{E}, i_{E^{\prime}}\right\rangle$. Then $E=C /\left\langle i_{E^{\prime}}\right\rangle$ and $E^{\prime}=C /\left\langle i_{E}\right\rangle$. It is easy to see that $Q_{11}^{C}, Q_{12}^{C}$ are the ramification points of the natural map $\pi_{E^{\prime}}: C \rightarrow E^{\prime}$ and $Q_{51}^{C}, Q_{52}^{C}$ are the ramification points of $\pi_{E}: C \rightarrow E$. In particular $i_{E}\left(Q_{1 j}^{C}\right)=Q_{1 j}^{C}, j=1,2 ; i_{E}\left(Q_{i 1}^{C}\right)=Q_{i 2}^{C}, i=2,3,4,5 ; i_{E^{\prime}}\left(Q_{5 j}^{C}\right)=Q_{5 j}^{C}$, $j=1,2 ; i_{E^{\prime}}\left(Q_{i 1}^{C}\right)=Q_{i 2}^{C}, i=1,2,3,4$. Then the six points $Q_{i j}^{C}$ where $i=2,3,4$ and $j=1,2$ are fixed by the involution $i_{E} i_{E^{\prime}}$ and $\pi_{\Gamma}: C \rightarrow \Gamma=C /\left\langle i_{E} i_{E^{\prime}}\right\rangle$ is the hyperelliptic map. Now we can find a basis $\left\langle\eta_{E}, \eta_{E^{\prime}}\right\rangle$ of $H^{0}\left(C, \Omega_{C}^{1}\right)$ where $\eta_{E}$ is a $i_{E^{\prime-}}$ invariant form and $\eta_{E^{\prime}}$ is $i_{E}$-invariant. If $G^{\star}=\left\{\mathrm{id}, \chi_{1}, \chi_{2}, \chi_{3}\right\}$ where $\chi_{1}\left(i_{E}\right)=-1$, $\chi_{1}\left(i_{E^{\prime}}\right)=1, \chi_{2}\left(i_{E}\right)=1, \chi_{2}\left(i_{E^{\prime}}\right)=-1, \chi_{1} \chi_{2}=\chi_{3}$ then $\left\langle\eta_{E}\right\rangle=V_{1, \chi_{1}},\left\langle\eta_{E^{\prime}}\right\rangle=V_{1, \chi_{2}}$. We have construct the $\mathbb{Z} / 2 \times \mathbb{Z} / 2$-action on the genus-2 curve $C=F$. We will construct the $\mathbb{Z} / 2 \times \mathbb{Z} / 2$-action on $D$ obtained in 3.7. We consider $\widetilde{E} \rightarrow \mathbb{P}^{1}=B$ and $\widetilde{E^{\prime}} \rightarrow \mathbb{P}^{1}=B$ two 2-to-1 morphisms branched on $P_{1}, \ldots P_{2 m}$ and on $A_{1}, A_{2}$ respectively and let $i_{\widetilde{E}}: \widetilde{E} \rightarrow \widetilde{E}, i_{\widetilde{E}^{\prime}}: \widetilde{E}^{\prime} \rightarrow \widetilde{E}^{\prime}$ be the natural involutions. Denote by $D$ the normalization of $\widetilde{E} \times{ }_{B} \widetilde{E}^{\prime}$; the natural map $\rho_{2}: D \rightarrow B$ is a $G=\mathbb{Z} / 2 \times \mathbb{Z} / 2$ - cover and $H^{0}\left(D, \Omega_{D}^{1}\right)=V_{1, \chi_{2}} \oplus V_{1, \chi_{3}}$. In fact it is easy to find a basis $\left\langle\alpha_{1}, \ldots, \alpha_{2 m-1}\right\rangle$ such that $\left\langle\alpha_{1}, \ldots, \alpha_{m-1}\right\rangle=V_{1, \chi_{2}}=H^{0}\left(\widetilde{E}, \Omega_{\widetilde{E}}^{1}\right)$, and $\left\langle\alpha_{m}, \ldots, \alpha_{2 m-1}\right\rangle=V_{2, \chi_{3}}=H^{0}\left(\widetilde{\Gamma}, \Omega_{\widetilde{\Gamma}}^{1}\right)$ where $\widetilde{\Gamma}=D /\left\langle i_{\widetilde{E}} i_{\widetilde{E}^{\prime}}\right\rangle$. The group $G=\left\langle\left(i_{E}, i_{\widetilde{E}}\right),\left(i_{E^{\prime}}, i_{\widetilde{E}^{\prime}}\right)\right\rangle$ acts diagonally on the product surface $Z=F \times D$ and if $X=Z / G, H^{0}\left(X, \Omega_{X}^{1}\right)=H^{0}\left(Z, \Omega_{Z}^{1}\right)^{G}=0, H^{0}\left(X, \Omega_{X}^{2}\right)=$ $H^{0}\left(Z, \Omega_{Z}^{2}\right)^{G}=V_{1, \chi_{2}} \otimes V_{2, \chi_{2}}$; that is $H^{0}\left(X, \Omega_{X}^{2}\right) \simeq\left\langle\eta \wedge \alpha_{1}, \ldots \eta \wedge \alpha_{m-1}\right\rangle$. Then $\Phi_{\left|K_{X}\right|}$ yields a pencil with image the rational normal curve of degree $m-1$ and general fibre $F$. We remark that if $A_{i} \neq R_{j}, i=1,2, j=1, \ldots, 2 m$ we obtain the family $I$ ); if $A_{1}=R_{1}$ and $A_{2} \neq R_{j} j=1, \ldots, 2 m$, we have $\left.I I\right)$; finally, if $A_{1}=R_{1}, A_{2}=R_{2}$ then we have $I I I)$. We can carry on the explicit computation of the nodes occurring in each family. We recall that $\rho_{1}^{-1}\left(Q_{i}\right)=\left\{Q_{i 1}, Q_{i 2}\right\}, i=1, \ldots, 5$ and we set $\rho_{2}^{-1}\left(R_{s}\right)=\left\{R_{s 1}, R_{s 2}\right\}$, $\rho_{2}^{-1}\left(A_{r}\right)=\left\{A_{r 1}, A_{r 2}\right\}$ where $s=1, \ldots, 2 m, r=1,2$. Then $\left\langle i_{E}\right\rangle=G_{Q_{11}}=G_{Q_{12}}$, $\left\langle i_{E^{\prime}}\right\rangle=G_{Q_{51}}=G_{Q_{52}},\left\langle i_{E} i_{E^{\prime}}\right\rangle=G_{Q_{i 1}}=G_{Q_{i 2}}, i=2,3,4$. Now it easy to see that in $I)\left\langle i_{\widetilde{E}^{\prime}}\right\rangle=G_{A_{r t}}, r, t=1,2,\left\langle i_{\widetilde{E}}\right\rangle=G_{R_{s 1}}=G_{R_{s 2}}, s=1, \ldots, 2 m$ which implies $t=8+8 m$. In $I I):\left\langle i_{\widetilde{E}^{\prime}}\right\rangle=G_{A_{2 t}}, t=1,2,\left\langle i_{\widetilde{E}}\right\rangle=G_{R_{s 1}}=G_{R_{s 2}}, s=2, \ldots, 2 m$, $\left\langle i_{\widetilde{E}^{\prime}} i_{\widetilde{E}}\right\rangle=G_{R_{11}}=G_{R_{12}}, t=12+8 m$. In $\left.I I I\right):\left\langle i_{\widetilde{E}}\right\rangle=G_{R_{s 1}}=G_{R_{s 2}}, s=3, \ldots, 2 m$, $\left\langle i_{\widetilde{E}^{\prime}} i_{\widetilde{E}}\right\rangle=G_{R_{11}}=G_{R_{12}}=G_{R_{21}}=G_{R_{22}}, t=16+8 \mathrm{~m}$

\section{$4 \quad g(F)=3$}

The classification of infinite families of $G$-sandwich canonically 3 -fibred surfaces is more difficult than the genus -2 case. Moreover there are many new cases with a rich structure. The following lemma is the analogue of 3.2 :

Lemma 4.1 Let $G$ be an Abelian group acting on a smooth genus-3 curve $F$ with quotient $A$. Let $\eta \in H^{0}\left(F, \Omega_{F}^{1}\right)$ be a 1-form such that $\operatorname{div}(\eta)$ is a $G$-invariant divisor and let $H^{0}\left(F, \Omega_{F}^{1}\right)=\oplus_{\chi \in G^{\star}} V_{1, \chi}$ be the decompositions into subspaces where $G$ acts on 
$V_{1, \chi}$ via the character $\chi$. Then the following cases occur:

\begin{tabular}{|l|c|l|}
\hline$g(A)$ & $G$ & $H^{0}\left(F, \Omega_{F}^{1}\right)$ \\
\hline 2 & $\mathbb{Z} / 2$ & $V_{1, \text { id }} \oplus V_{1, \chi}$ \\
\hline 1 & $\mathbb{Z} / 2$ & $V_{1, \text { id }} \oplus V_{1, \chi}$ \\
\hline 1 & $\mathbb{Z} / 3$ & $V_{1, \text { id }} \oplus V_{1, \chi} \oplus V_{1, \chi^{2}}$ \\
\hline 1 & $\mathbb{Z} / 4$ & $V_{1, \text { id }} \oplus V_{1, \chi} \oplus V_{1, \chi^{3}}$ \\
\hline 1 & $\mathbb{Z} / 2 \times \mathbb{Z} / 2$ & $V_{1, \text { id }} \oplus V_{1, \chi_{1}} \oplus V_{1, \chi_{2}}$ \\
\hline 0 & $\mathbb{Z} / 2$ & $V_{1, \chi}$ \\
\hline 0 & $\mathbb{Z} / 3$ & $V_{1, \chi} \oplus V_{1, \chi^{2}}$ \\
\hline 0 & $\mathbb{Z} / 4$ & $V_{1, \chi} \oplus V_{1, \chi^{2}} \oplus V_{1, \chi^{3}}$ \\
\hline 0 & $\mathbb{Z} / 4$ & $V_{1, \chi} \oplus V_{1, \chi^{3}}$ \\
\hline 0 & $\mathbb{Z} / 2 \times \mathbb{Z} / 2$ & $V_{1, \chi_{1}} \oplus V_{1, \chi_{1} \chi_{2}} \oplus V_{1, \chi_{2}}$ \\
\hline 0 & $\mathbb{Z} / 2 \times \mathbb{Z} / 2$ & $V_{1, \chi_{1}} \oplus V_{1, \chi_{2}}$ \\
\hline 0 & $\mathbb{Z} / 6$ & $V_{1, \chi} \oplus V_{1, \chi^{3}} \oplus V_{1, \chi^{5}}$ \\
\hline 0 & $\mathbb{Z} / 6$ & $V_{1, \chi} \oplus V_{1, \chi^{4}} \oplus V_{1, \chi^{5}}$ \\
\hline 0 & $\mathbb{Z} / 7$ & $V_{1, \chi} \oplus V_{1, \chi^{2}} \oplus V_{1, \chi^{4}}$ \\
\hline 0 & $\mathbb{Z} / 7$ & $V_{1, \chi} \oplus V_{1, \chi^{2}} \oplus V_{1, \chi^{5}}$ \\
\hline 0 & $\mathbb{Z} / 8$ & $V_{1, \chi} \oplus V_{1, \chi^{2}} \oplus V_{1, \chi^{5}}$ \\
\hline 0 & $\mathbb{Z} / 8$ & $V_{1, \chi} \oplus V_{1, \chi^{2}} \oplus V_{1, \chi^{3}}$ \\
\hline 0 & $\mathbb{Z} / 2 \times \mathbb{Z} / 4$ & $V_{1, \chi_{1} \chi_{2}} \oplus V_{1, \chi_{1} \chi_{2}^{2}} \oplus V_{1, \chi_{2}}$ \\
\hline 0 & $\mathbb{Z} / 2 \times \mathbb{Z} / 4$ & $V_{1, \chi_{1} \chi_{2}} \oplus V_{1, \chi_{1} \chi_{2}^{2}} \oplus V_{1, \chi_{2}^{3}}$ \\
\hline 0 & $\mathbb{Z} / 2 \times \mathbb{Z} / 2 \times \mathbb{Z} / 2$ & $V_{1, \chi_{100}} \oplus V_{1, \chi_{010}} \oplus V_{1, \chi_{001}}$ \\
\hline 0 & $\mathbb{Z} / 9$ & $V_{1, \chi} \oplus V_{1, \chi^{2}} \oplus V_{1, \chi^{4}}$ \\
\hline 0 & $\mathbb{Z} / 12$ & $V_{1, \chi} \oplus V_{1, \chi^{2}} \oplus V_{1, \chi^{5}}$ \\
\hline 0 & $\mathbb{Z} / 14$ & $V_{1, \chi} \oplus V_{1, \chi^{3}} \oplus V_{1, \chi^{5}}$ \\
\hline 0 & $\mathbb{Z} / 2 \times \mathbb{Z} / 8$ & $V_{1, \chi_{2}^{3}} \oplus V_{1, \chi_{1} \chi_{2}^{2}} \oplus V_{1, \chi_{2}}$ \\
\hline 0 & $\mathbb{Z} / 4 \times \mathbb{Z} / 4$ & $V_{1, \chi_{1}} \oplus V_{1, \chi_{1}^{2} \chi_{2}^{3}} \oplus V_{1, \chi_{2}}$ \\
\hline
\end{tabular}

where $\chi$ is a generator of $(\mathbb{Z} / d)^{\star}$ if $d=2,3,4,6,7,8,9,12, \chi_{1}, \chi_{2}$ generate $(\mathbb{Z} / a \times \mathbb{Z} / b)^{\star}$ if $(a, b)=(2,2),(2,4),(2,7),(2,8),(4,4)$ and $\chi_{100}, \chi_{010}, \chi_{001}$ generate $(\mathbb{Z} / 2 \times \mathbb{Z} / 2 \times$ $\mathbb{Z} / 2)^{\star}$.

Proof. The proof is straightforward but long. It requires two basic ingredients: a standard Hurwitz's argument, plus the realization of $G$ as a $\Gamma$-quotient via a homomorphism with torsion free kernel, where $\Gamma=\left\langle a_{1}, b_{1}, \ldots, a_{b}, b_{b}, x_{1}, \ldots, x_{m}\right| \prod_{i=1}^{b}\left[a_{i}, b_{i}\right] x_{1} \cdots x_{m}=$ $\left.x_{1}^{e_{1}}=\cdots x_{m}^{e_{m}}=1\right\rangle, 0 \leq b \leq 2, e_{i}$, is the ramification index of each $P_{i j} \in \pi^{-1}\left(Q_{i}\right)$ and $Q_{1}, \ldots, Q_{m} \in A$ are the branch points of $\pi: F \rightarrow A=F / G . \quad \dashv$

We consider a surface $S$ with $p_{g} \gg 0$ and genus-3 fibre of the canonical pencil $f$ : $S \rightarrow B$. We call $b$ the genus of $B$ and $q=q(S)$ the irregularity of $S$. From 2.5 we have to consider the last four cases in the table (3): $A_{0}^{(3)}$ i.e. $q=b=0 ; A_{1}^{(3)}$ i.e. $q=1$, 
$b=0 ; A_{2}^{(3)}$ i.e. $q=2, b=0 ; B^{(3)}$ i.e. $q=b=1$. We divide our classification-proof into two parts: $|G| \leq 8$ and $|G|>8$

\section{1 $|G| \leq 8$}

The examples of surfaces with isotrivial canonical pencil with genus-3 fibre and $p_{g} \gg$ 0 existing in the literature, as fas as we know, are $G$-sandwich canonically 3 -fibred surfaces with a $\mathbb{Z} / 2$-action or a $\mathbb{Z} / 2 \times \mathbb{Z} / 2$-action. As a by-product of our classification we will see that all $G$-sandwich canonically 3 -fibred surfaces with $p_{g} \gg 0$ and $|G| \leq 8$ have $G=\mathbb{Z} / 2$ or $G=\mathbb{Z} / 2 \times \mathbb{Z} / 2$ or $G=\mathbb{Z} / 2 \times \mathbb{Z} / 2 \times \mathbb{Z} / 2$.

$\mathbf{q}=\mathbf{b}=\mathbf{0}, \mathbf{g}=\mathbf{3}$ : The surfaces constructed in this section are all new. In fact the case $q=b=0$ is the richer and the harder one.

Proposition 4.2 If $S$ is a G-sandwich canonically 3-fibred surface on a rational curve with $|G| \leq 8, q(S)=0, p_{g}(S)=m-1 \geq 3$ then $S$ is the minimal desingularization of $X=Z / G$ where $G$ acts diagonally on $Z=F \times D, g(F)=3, X$ has $t$ nodes and $S$ is in one of the following classes:

Case $G=\mathbb{Z} / 2 \times \mathbb{Z} / 2$ :

$$
\begin{array}{rlll}
I) & g(D)=2 m-1 & K_{S}^{2}=8 \chi\left(\mathcal{O}_{S}\right) & t=16 \\
I I) & g(D)=2 m-2 & K_{S}^{2}=8 \chi\left(\mathcal{O}_{S}\right)-12 & t=24 \\
I I I) & g(D)=2 m-3 & K_{S}^{2}=8 \chi\left(\mathcal{O}_{S}\right)-16 & t=32
\end{array}
$$

Case $G=\mathbb{Z} / 2 \times \mathbb{Z} / 2 \times \mathbb{Z} / 2$ :

$$
\begin{array}{rlll}
\text { i) } & g(D)=4 m+1 & K_{S}^{2}=8 \chi\left(\mathcal{O}_{S}\right) & t=0 \\
\text { ii) } & g(D)=4 m-1 & K_{S}^{2}=8 \chi\left(\mathcal{O}_{S}\right)-4 & t=16 \\
\text { iii) } & g(D)=4 m-3 & K_{S}^{2}=8 \chi\left(\mathcal{O}_{S}\right)-8 & t=32 \\
\text { iv) } & g(D)=4 m-5 & K_{S}^{2}=8 \chi\left(\mathcal{O}_{S}\right)-12 & t=48 \\
\text { v) } & g(D)=4 m-7 & K_{S}^{2}=8 \chi\left(\mathcal{O}_{S}\right)-16 & t=64
\end{array}
$$

Proof. We have to consider groups $G$ in table (7) with $|G| \leq 8$ and $g(A)=0$. In fact we have to classify all the $G$ coverings $D \rightarrow D / G=B \simeq \mathbb{P}^{1}$ such that the induced decomposition $H^{0}\left(D, \Omega_{D}^{1}\right)=\oplus_{\chi \in G^{\star}} V_{2, \chi}$ satisfies the two conditions in 2.3 . Now $G=\mathbb{Z} / 2$ does not occur since $\operatorname{dim} V_{1, \text { id }}=0, \operatorname{dim} V_{2, \text { id }}=0$ and $\operatorname{dim} V_{1,- \text { id }}=3$, $\operatorname{dim} V_{2,- \text { id }}>1$. $G=\mathbb{Z} / 3$ does not occur. In fact we have to solve the system (5) which becomes a simple system with integer coefficients; we can assume $\operatorname{dim} V_{2, \chi^{2}}=0$ where $G^{\star}=\langle\chi\rangle$. Then it leads to the following solution: $1=\operatorname{dim} V_{2, \chi}=g(D)=p_{g}(S)$; $0=\operatorname{dim} V_{2, \chi}=g(D)=p_{g}(S)$; which corresponds to a surface not of general type. By the general theory of Abelian covers $D \rightarrow B=D / \mathbb{Z} / 4$ is given through $\mathcal{L}_{\chi}, \mathcal{L}_{\chi^{2}}, \mathcal{L}_{\chi^{3}} \in$ $\operatorname{Pic}(B)$ and three effective divisors $D_{1}, D_{2}, D_{3}$ such that: 


$$
\left\{\begin{aligned}
4 \mathcal{L}_{\chi} & =D_{1}+2 D_{2}+3 D_{3} \\
\mathcal{L}_{\chi^{2}} & =2 \mathcal{L}_{\chi}-D_{2}-D_{3} \\
\mathcal{L}_{\chi^{3}} & =3 \mathcal{L}_{\chi}-D_{2}-2 D_{3} .
\end{aligned}\right.
$$

We have to consider the two $\mathbb{Z} / 4$-action in the table (7). If $H^{0}\left(F, \Omega_{F}^{1}\right)=V_{1, \chi} \oplus V_{1, \chi^{2}} \oplus$ $V_{1, \chi^{3}}$, then we can assume $\operatorname{dim} V_{2, \chi^{3}}=0$ that is $\operatorname{deg}\left(\mathcal{L}_{\chi^{3}}\right)=1$. Since $D \rightarrow D / G=B$ is connected we have $\operatorname{deg}\left(\mathcal{L}_{\chi^{2}}\right)>0$ and $\operatorname{deg}\left(\mathcal{L}_{\chi^{1}}\right)>0$. Then $g(D) \leq 1$ i.e. $S$ is not of general type. If $H^{0}\left(F, \Omega_{F}^{1}\right)=V_{1, \chi} \oplus V_{1, \chi^{3}}$ with $\operatorname{dim} V_{1, \chi}=2$ then $\operatorname{dim} V_{2, \chi^{3}}=0$. Moreover $\operatorname{deg}\left(\mathcal{L}_{\chi^{2}}\right)>0$. Then we obtain $\left.i\right) \operatorname{deg}\left(\mathcal{L}_{\chi^{2}}\right)=1, \operatorname{deg}\left(\mathcal{L}_{\chi}\right)=2$ or $\left.i i\right) \operatorname{deg}\left(\mathcal{L}_{\chi^{2}}\right)=2$, $\operatorname{deg}\left(\mathcal{L}_{\chi}\right)=3$. Then $g(D)=3$ and the minimal desingularization $S$ has $p_{g}=2$.

The case $G=\mathbb{Z} / 2 \times \mathbb{Z} / 2$.

We recall that the stabilizer $G(P)$ of a point $P \in Z$ is the trivial group or $G(P)=\mathbb{Z} / 2$. In particular by 2.6 the minimal resolution $S \rightarrow X=Z / G$ is the blow up of the $t$ nodes of $X$ and $S$ is minimal. We want three reduced divisors $D_{1}, D_{2}, D_{3}$ and three line bundles on $\mathbb{P}^{1}, \mathcal{L}_{\chi_{1}} \mathcal{L}_{\chi_{2}} \mathcal{L}_{\chi_{1} \chi_{2}}$ which satisfy the system (6). Set $m=\operatorname{deg}\left(\mathcal{L}_{\chi_{1}}\right)$ and we easily see that we obtain the desired solutions.

If $G=\mathbb{Z} / 6=\mathbb{Z} / 3 \times \mathbb{Z} / 2=H \times K, G^{\star}=\langle\chi\rangle, H^{\star}=\langle\phi\rangle, K^{\star}=\langle\psi\rangle$ where $\chi_{\mid H}=\phi$ and $\chi_{\mid K}=\psi$ by [11] [Proposition 2.1 and 2.15]we have to solve

$$
\left\{\begin{array}{l}
6 \mathcal{L}_{\chi}=D_{\chi}+2 D_{\phi}+4 D_{\phi^{2}}+3 D_{\psi}+5 D_{\chi^{-1}} \\
\mathcal{L}_{\chi^{2}}=2 \mathcal{L}_{\chi}-D_{\phi^{2}}-D_{\psi}-D_{\chi^{-1}} \\
\mathcal{L}_{\chi^{3}}=3 \mathcal{L}_{\chi}-D_{\phi}-D_{\phi^{2}}-D_{\psi}-2 D_{\chi^{-1}} \\
\mathcal{L}_{\chi^{4}}=4 \mathcal{L}_{\chi}-D_{\phi}-2 D_{\phi^{2}}-2 D_{\psi}-3 D_{\chi^{-1}} \\
\mathcal{L}_{\chi^{5}}=5 \mathcal{L}_{\chi}-D_{\phi}-3 D_{\phi^{2}}-2 D_{\psi}-4 D_{\chi^{-1}}
\end{array}\right.
$$

Looking to the two actions in 7 we easily see that we can assume that $V_{2, \chi^{5}}=0$ that is $\operatorname{deg}\left(\mathcal{L}_{\chi}\right)=1$. Then it is obvious that $p_{g}(S)$ is small. A brute computation shows that if $H^{0}\left(F, \Omega_{F}^{1}\right)=V_{1, \chi} \oplus V_{1, \chi^{3}} \oplus V_{1, \chi^{5}}$ then $H^{0}\left(D, \Omega_{D}^{1}\right)=V_{2, \chi} \oplus V_{2, \chi^{2}} \oplus V_{1, \chi^{4}}$ with $\operatorname{dim} V_{2, \chi}=2$ $\operatorname{dim} V_{2, \chi^{2}}=\operatorname{dim} V_{2, \chi^{4}}=1, g(D)=4, p_{g}(S)=2$ and if $H^{0}\left(F, \Omega_{F}^{1}\right)=V_{1, \chi} \oplus V_{1, \chi^{2}} \oplus V_{1, \chi^{5}}$ then $H^{0}\left(D, \Omega_{D}^{1}\right)=V_{2, \chi} \oplus V_{2, \chi^{2}} \oplus V_{1, \chi^{3}}$ with $\operatorname{dim} V_{2, \chi}=\operatorname{dim} V_{2, \chi^{2}}=\operatorname{dim} V_{2, \chi^{3}}=1$, $g(D)=3, p_{g}(S)=1$ or $H^{0}\left(D, \Omega_{D}^{1}\right)=V_{2, \chi} \oplus V_{2, \chi^{2}} \oplus V_{1, \chi^{3}}$ with $\operatorname{dim} V_{2, \chi}=2 \operatorname{dim} V_{2, \chi^{2}}=$ $\operatorname{dim} V_{2, \chi^{3}}=1, g(D)=4, p_{g}(S)=2$.

The cases $G=\mathbb{Z} / 7$ or $G=\mathbb{Z} / 8$ or $G=\mathbb{Z} / 2 \times \mathbb{Z} / 4$ have no solutions.

The case $G=\mathbb{Z} / 2 \times \mathbb{Z} / 2 \times \mathbb{Z} / 2$ :

Let $G=H_{100} \times H_{010} \times H_{001}, G^{\star}=\left\{\chi_{i j k}\right\} \cup\{$ id $\}$ where $H_{i j k} \simeq \mathbb{Z} / 2$ are the 7 non-trivial subgroups and $\chi_{i j k}$ are the non-trivial characters of $G$ with the obvious notation. We want divisors $D_{i j k}$ and line bundles $L_{i j k}$ on $\mathbb{P}^{1}$ such that 


$$
\left\{\begin{aligned}
2 \mathcal{L}_{100} & =D_{100}+D_{110}+D_{101}+D_{111} \\
2 \mathcal{L}_{010} & =D_{010}+D_{110}+D_{011}+D_{111} \\
2 \mathcal{L}_{001} & =D_{001}+D_{101}+D_{011}+D_{111} \\
\mathcal{L}_{110} & =\mathcal{L}_{100}+\mathcal{L}_{010}-D_{110}-D_{111} \\
\mathcal{L}_{101} & =\mathcal{L}_{100}+\mathcal{L}_{001}-D_{101}-D_{111} \\
\mathcal{L}_{011} & =\mathcal{L}_{010}+\mathcal{L}_{001}-D_{011}-D_{111} \\
\mathcal{L}_{111} & =\mathcal{L}_{100}+\mathcal{L}_{010}+\mathcal{L}_{001}-D_{110}-D_{101}-D_{011}-D_{111}
\end{aligned}\right.
$$

Set $d_{i j k}^{1}=\operatorname{deg} D_{i j k}$ in the case of a $G$-action on $F$ and $d_{i j k}^{2}=\operatorname{deg} D_{i j k}$ in the case of a $G$-action on $D$. We recall that $d_{i j k}^{s}$ is the number of points $Q$ on $C_{s}$ such that the stabilizer $G_{P}=H_{i j k}$ where $P \in \pi_{s}^{-1}(Q)$ and $\pi_{s}: C_{s}^{\prime} \rightarrow C_{s}$ is the $\mathbb{Z} / 2 \times \mathbb{Z} / 2 \times \mathbb{Z} / 2$ Galois cover, $s=1,2$. The $G$-action on a genus-3 curve $F$ with $\pi: F \rightarrow F / G=A=$ $\mathbb{P}^{1}$ is unique and it is described by the following numbers: $d_{100}^{1}=d_{010}^{1}=d_{001}^{1}=0$ $d_{110}^{1}=d_{011}^{1}=d_{101}^{1}=1, d_{111}^{1}=2$. By table (7) and theorem 2.3 we can assume $\operatorname{deg} \mathcal{L}_{010}=\operatorname{deg} \mathcal{L}_{001}=1$ in the system (10). Moreover since $D$ is connected $\operatorname{deg} \mathcal{L}_{i j k} \geq 1$ for every triple $(i, j, k)$. Then we can easily solve (10) and the desired $G$-actions on a curve $D$ are:

i) $d_{111}^{2}=d_{011}^{2}=d_{101}^{2}=d_{110}^{2}=0$

ii) $d_{111}^{2}=d_{011}^{2}=d_{101}^{2}=0$,

$d_{010}^{2}=d_{001}^{2}=2$

$d_{100}^{2}=2 m$

iii) $d_{111}^{2}=d_{011}^{2}=0$,

$d_{110}^{2}=d_{010}^{2}=1, d_{001}^{2}=2$,

$d_{100}^{2}=2 m-1$

iv) $d_{111}^{2}=d_{010}^{2}=d_{001}^{2}=0$,

$d_{101}^{2}=d_{110}^{2}=d_{010}^{2}=d_{001}^{2}=1$,

$d_{100}^{2}=2 m-2$

v) $d_{111}^{2}=d_{010}^{2}=d_{001}^{2}=d_{011}^{2}=0$,

$d_{110}^{2}=d_{101}^{2}=d_{011}^{2}=1$,

$d_{100}^{2}=2 m-2$

$d_{110}^{2}=d_{101}^{2}=2$,

$d_{100}^{2}=2 m-4 . \dashv$

\subsubsection{A geometrical construction of the families of 4.2}

We will show a geometrical procedure to construct the families found in 4.2 .

The case $G=\mathbb{Z} / 2 \times \mathbb{Z} / 2$ :

we start with $A=B=\mathbb{P}^{1}$. Let $\pi: C \rightarrow \mathbb{P}^{1}$ the $2: 1$-cover induced by the hyperelliptic involution $j_{1}: C \rightarrow C$ on a genus-2 curve and let $W_{1}, \ldots, W_{6} \in C$ be the Weierstrass points. Set $\pi\left(W_{i}\right)=A_{i} i=1, \ldots, 6$. Let $E \rightarrow \mathbb{P}^{1}$ the $2: 1$-cover branched on $A_{1}, A_{2}, A_{3}, A_{4}$ and $i_{1}: E \rightarrow E$ the induced involution on the elliptic curve $E$. The group $\left\langle i_{1}\right\rangle \times\left\langle j_{1}\right\rangle \simeq \mathbb{Z} / 2 \times \mathbb{Z} / 2$ acts on the fibre product $C \times_{\mathbb{P}^{1}} E=F$ in the obvious way, $\Gamma=F /\left\langle i_{1} j_{1}\right\rangle$ is the $2: 1$-cover $\Gamma \rightarrow \mathbb{P}^{1}$ branched on $A_{5}, A_{6}, F /\left\langle j_{1}\right\rangle=E$, $F /\left\langle i_{1}\right\rangle=C$. Let $G^{\star}=\left\{\right.$ id, $\left.\chi_{1}, \chi_{2}, \chi_{3}\right\}$ where $\chi_{3}=\chi_{1} \circ \chi_{2} \chi_{1}\left(i_{1}\right)=-1, \chi_{1}\left(j_{1}\right)=1$, $\chi_{2}\left(i_{1}\right)=1, \chi_{2}\left(j_{1}\right)=-1$ then $H^{0}\left(F, \Omega_{F}^{1}\right)=V_{1, \chi_{1}} \oplus V_{1, \chi_{2}}$ where $V_{1, \chi_{1}}=\langle\eta\rangle \simeq H^{0}\left(E, \Omega_{E}^{1}\right)$, $V_{1, \chi_{2}}=\left\langle\mu_{1}, \mu_{2}\right\rangle \simeq H^{0}\left(C, \Omega_{C}^{1}\right)$. Let $\widetilde{\pi}: \widetilde{C} \rightarrow \mathbb{P}^{1}, \widetilde{E} \rightarrow \mathbb{P}^{1}$ be two $2: 1$-cover branched respectively on $R_{1}, \ldots, R_{2 m}$ and on $Q_{1}, Q_{2}$; then $g(\widetilde{C})=m-1, g(\widetilde{E})=0$. Let $i_{2}: \widetilde{C} \rightarrow \widetilde{C}, j_{2}: \widetilde{E} \rightarrow \widetilde{E}$ the induced involutions. The group $\left\langle i_{2}\right\rangle \times\left\langle j_{2}\right\rangle$ acts on the fibre product $\widetilde{C} \times \mathbb{P}^{1} \widetilde{E}=D$ and $\widetilde{\Gamma}=F /\left\langle i_{2} j_{2}\right\rangle$ is the $2: 1$-cover $\widetilde{\Gamma} \rightarrow \mathbb{P}^{1}$ branched on $Q_{1}, Q_{2}, R_{1}, \ldots R_{2 m}$, then $g(\widetilde{\Gamma})=m$ and $D \rightarrow \widetilde{\Gamma}$ is unramified. In particular $H^{0}\left(D, \Omega_{D}^{1}\right)=$ $V_{2, \chi_{1}} \oplus V_{2, \chi_{3}}$ where $V_{2, \chi_{1}}=\left\langle\alpha_{1}, \ldots \alpha_{m-1}\right\rangle \simeq H^{0}\left(\widetilde{C}, \Omega_{\widetilde{C}}^{1}\right), V_{2, \chi_{3}}=\left\langle\beta_{1}, \ldots, \beta_{m}\right\rangle \simeq H^{0}\left(\widetilde{\Gamma}, \Omega_{\widetilde{\Gamma}}^{1}\right)$. 
Consider $Z=F \times D$ with the $G$-action given by $\langle i, j\rangle$ where $i=\left(i_{1}, i_{2}\right), j=\left(j_{1}, j_{2}\right)$. In particular on $X=Z / G$ there are the 16 nodes obtained by the 16 points of $Z$ with stabilizer $\langle j\rangle$. Since $\left.H^{0}\left(X, \Omega_{X}^{2}\right)=H^{0}\left(Z, \Omega_{Z}^{2}\right)^{G}=\left[\left(V_{1, \chi_{1}} \oplus V_{1, \chi_{2}}\right) \otimes V_{2, \chi_{1}} \oplus V_{2, \chi_{3}}\right)\right]^{G}$ then $\left.H^{0}\left(X, \Omega_{X}^{2}\right)=V_{1, \chi_{1}} \otimes V_{2, \chi_{1}}\right)=\left\{\eta \wedge \alpha_{1}, \ldots, \eta \wedge \alpha_{m-1}\right\}$; that is $X$ is the family $\left.I\right)$ of 4.2 . The families $I I)$ and $I I I)$ are obtained by a degeneration argument. In fact if the point $R_{1}$ coincides with $Q_{1}$ we have $\left.I I\right)$ while $\left.I I I\right)$ is given by the conditions: $R_{1}=Q_{1}$ and $R_{2}=Q_{2}$.

The case $G=\mathbb{Z} / 2 \times \mathbb{Z} / 2 \times \mathbb{Z} / 2$

We consider the $\mathbb{Z} / 2 \times \mathbb{Z} / 2$-action on a genus- 2 curve $C$ which gives the three families of $G$-sandwich canonically 2 -fibred surfaces on a rational curve with $q(S)=0$ described in section 3.1. With the same notation there let $\rho_{E^{\prime \prime}}: E^{\prime \prime} \rightarrow A=\mathbb{P}^{1}$ be the $\mathbb{Z} / 2$-cover branched on $Q_{2}, Q_{3}$ and let $i_{E^{\prime \prime}}: E^{\prime \prime} \rightarrow E^{\prime \prime}$ be the corresponding involution. The group $\left\langle i_{E^{\prime \prime}}\right\rangle \times\left\langle i_{E}\right\rangle \times\left\langle i_{E^{\prime}}\right\rangle$ acts on the normalization of the fibre product $F=E^{\prime \prime} \times_{A} C=$ $E^{\prime \prime} \times_{A} E \times_{A} E^{\prime}$ and let $\pi_{F} F \rightarrow A$ be the $\mathbb{Z} / 2 \times \mathbb{Z} / 2 \times \mathbb{Z} / 2$-Galois cover. We set $\pi_{F}^{-1}\left(Q_{i}\right)=\left\{Q_{i 1}, Q_{i 2}, Q_{i 3}, Q_{i 4}\right\}$, in particular $\pi_{F}^{\star}\left(Q_{i}\right)=2 Q_{i 1}+2 Q_{i 2}+2 Q_{i 3}+2 Q_{i 4}$. It is easy to see that $C=F /\left\langle i_{E^{\prime \prime}}\right\rangle, C^{\prime}=F /\left\langle i_{E}\right\rangle=E^{\prime \prime} \times_{A} E$ and $C^{\prime \prime}=F /\left\langle i_{E^{\prime}}\right\rangle=E^{\prime \prime} \times_{A} E^{\prime}$ are genus- 2 curves and that $Q_{1}=D_{101}^{1}, Q_{2}+Q_{3}=D_{111}^{1}, Q_{4}=D_{011}^{1}, Q_{5}=D_{110}^{1}$. Moreover we can find a basis $\left\{\eta, \eta^{\prime}, \eta^{\prime \prime}\right\}$ of $H^{0}\left(F, \Omega_{F}^{1}\right)$ such that $\left\langle\eta^{\prime}, \eta^{\prime \prime}\right\rangle=H^{0}\left(C, \Omega_{C}^{1}\right),\left\langle\eta, \eta^{\prime \prime}\right\rangle=$ $H^{0}\left(C^{\prime}, \Omega_{C^{\prime}}^{1}\right),\left\langle\eta, \eta^{\prime}\right\rangle=H^{0}\left(C^{\prime \prime}, \Omega_{C^{\prime \prime}}^{1}\right)$. Then $V_{1, \chi_{100}}=\langle\eta\rangle, V_{1, \chi_{010}}=\left\langle\eta^{\prime}\right\rangle, V_{1, \chi_{001}}=\left\langle\eta^{\prime \prime}\right\rangle$. We have described the $\mathbb{Z} / 2 \times \mathbb{Z} / 2 \times \mathbb{Z} / 2$-action on $F$. We will construct the suitable $G$-action on $D$. Let $B \rightarrow B, B^{\prime} \rightarrow B, B^{\prime \prime} \rightarrow B$ be the $\mathbb{Z} / 2$-covers of $B=\mathbb{P}^{1}$ branched on $\left\{A_{1}^{010}, A_{2}^{010}\right\},\left\{A_{1}^{001}, A_{2}^{001}\right\}$ and $\left\{A_{1}^{100}, \ldots, A_{2 m}^{100}\right\}$ respectively and $i_{100}: B^{\prime \prime} \rightarrow B^{\prime \prime}$, $i_{010}: B \rightarrow B, i_{001}: B^{\prime} \rightarrow B^{\prime}$ the corresponding involutions. Let $D=B^{\prime \prime} \times_{B} B \times_{B} B^{\prime}$ then $G=\left\langle i_{100}, i_{010}, i_{001}\right\rangle$ acts on $D$. We set $C_{100}=D /\left\langle i_{100}\right\rangle, C_{010}=D /\left\langle i_{010}\right\rangle, C_{001}=$ $D /\left\langle i_{001}\right\rangle$. Then $C_{100}$ is elliptic and $C_{100} \rightarrow B$ is branched on $\left\{A_{1}^{010}, A_{2}^{010}, A_{1}^{001}, A_{2}^{001}\right\}$ while $g\left(C_{010}\right)=g\left(C_{001}\right)=2 m-1$ and $C_{010} \rightarrow B, C_{001} \rightarrow B$ have branch locus respectively $\left\{A_{1}^{001}, A_{2}^{001}\right\} \cup\left\{A_{1}^{100}, \ldots, A_{2 m}^{100}\right\}$ and $\left\{A_{1}^{010}, A_{2}^{010}\right\} \cup\left\{A_{1}^{100}, \ldots, A_{2 m}^{100}\right\}$. We can find a basis $\langle\epsilon\rangle \cup\left\langle\alpha_{i}\right\rangle_{i=1, \ldots, m-1} \cup\left\langle\beta_{j}\right\rangle_{j=1, \ldots, m} \cup\left\langle\gamma_{r}\right\rangle_{r=1, \ldots, m} \cup\left\langle\delta_{s}\right\rangle_{s=1, \ldots, m+1}$ of $H^{0}\left(D, \Omega_{D}^{1}\right)$ such that $G$ acts in the following way:

\begin{tabular}{|l|l|l|l|}
\hline & $i_{100}^{\star}$ & $i_{010}^{\star}$ & $i_{001}^{\star}$ \\
\hline$\epsilon$ & $\epsilon$ & $-\epsilon$ & $-\epsilon$ \\
\hline$\alpha$ & $-\alpha$ & $\alpha$ & $\alpha$ \\
\hline$\beta$ & $-\beta$ & $\beta$ & $-\beta$ \\
\hline$\gamma$ & $-\gamma$ & $-\gamma$ & $\gamma$ \\
\hline$\delta$ & $-\delta$ & $-\delta$ & $-\delta$ \\
\hline
\end{tabular}

in particular $V_{2, \chi_{100}}=\left\langle\alpha_{1}, \ldots, \alpha_{m-1}\right\rangle, V_{2, \chi_{010}}=0, V_{2, \chi_{001}}=0$. Now it is easy to construct a $G=\mathbb{Z} / 2 \times \mathbb{Z} / 2 \times \mathbb{Z} / 2$-action on the product $Z=F \times D$ such that $X=Z / G$, $\left.H^{0}\left(X, \Omega_{X}^{2}\right)=H^{0}\left(Z, \Omega_{Z}^{2}\right)^{G}=V_{1, \chi_{100}} \otimes V_{2, \chi_{100}}\right)=\left\{\eta \wedge \alpha_{1}, \ldots, \eta \wedge \alpha_{m-1}\right\}$ and $S=X / G$ is the surface with $p_{g}(S)=m-1$ of type $i$ ) in 4.2. We can obtain the other surfaces in 4.2 with a $\mathbb{Z} / 2 \times \mathbb{Z} / 2 \times \mathbb{Z} / 2$-action through a degeneration argument similar to the 
previous one in the $\mathbb{Z} / 2 \times \mathbb{Z} / 2$-case.

$q=1, b=0, g=3$ : as far as we know the only examples of isotrivial canonical fibrations with $q=1, b=0, g=3$ are in [20], see also [5] and [6]. Here we will give the complete classification of $G$-sandwich canonically 3 -fibred surfaces on a rational curve with $p_{g}(S) \gg 0, q=1$.

Proposition 4.3 If $S$ is a $G$-sandwich canonically 3 -fibred surface on a rational curve with $q(S)=1, p_{g}(S)=m-1 \geq 2$ then $S$ is the minimal desingularization of $X=Z / G$ where $G=\mathbb{Z} / 2 \times \mathbb{Z} / 2$ acts diagonally on $Z=F \times D, g(F)=3$ and $S$ is in one of the following classes:

$$
\begin{array}{rlll}
\text { I) } & g(D)=2 m-1 & K_{S}^{2}=8 \chi\left(\mathcal{O}_{S}\right) & t=0 \\
I I) & g(D)=2 m-2 & K_{S}^{2}=8 \chi\left(\mathcal{O}_{S}\right)-4 & t=8 \\
\text { III) } & g(D)=2 m-3 & K_{S}^{2}=8 \chi\left(\mathcal{O}_{S}\right)-8 & t=16
\end{array}
$$

Proof. It is easier than the proof of 4.2. In fact $g(A)=1$ and by the table (7) we have to classify all the $G$-coverings $D \rightarrow D / G=B \simeq \mathbb{P}^{1}$ where $G=\mathbb{Z} / 2$ or $G=\mathbb{Z} / 3$ or $G=\mathbb{Z} / 4$ or $G=\mathbb{Z} / 2 \times \mathbb{Z} / 2$ such that the induced decomposition $H^{0}\left(D, \Omega_{D}^{1}\right)=\oplus_{\chi \in G^{\star}} V_{2, \chi}$ satisfies the two conditions in 2.3. We exclude $G=\mathbb{Z} / 2$, $G=\mathbb{Z} / 3, G=\mathbb{Z} / 4$ by a simple computation as in 4.2 . Assume $G=\mathbb{Z} / 2 \times \mathbb{Z} / 2$. We want three reduced divisors $D_{1}, D_{2}, D_{3}$ and three line bundles on $\mathbb{P}^{1}, \mathcal{L}_{\chi_{1}}, \mathcal{L}_{\chi_{2}}, \mathcal{L}_{\chi_{1} \chi_{2}}$ under the constraints $\operatorname{deg}\left(\mathcal{L}_{\chi_{2}}\right)=1, \operatorname{deg}\left(\mathcal{L}_{\chi_{1} \chi_{2}}\right) \geq 1$. Set $m=\operatorname{deg}\left(\mathcal{L}_{\chi_{1}}\right)$ and we easily solve the system (6) which gives the desired solutions $\dashv$

\subsubsection{The three families of proposition 4.3: a geometrical construction}

We want to construct a $\mathbb{Z} / 2 \times \mathbb{Z} / 2$-cover $\pi_{1}: F \rightarrow A$ on the elliptic curve $A$. Let $C \rightarrow A$ be the 2-to-1 cover branched on $P, Q \in A, \pi_{1}^{-1}(P)=P_{1}, \pi_{1}^{-1}(Q)=Q_{1}$ and set $C^{\prime}$ another copy of $C$; call $i_{C}: C \rightarrow C$ and $i_{C^{\prime}}: C^{\prime} \rightarrow C^{\prime}$ the associated involutions, let $\nu: F \rightarrow C \times{ }_{A} C^{\prime}$ be the normalization map and set $\left\{P_{1}^{1}, P_{1}^{2}\right\}=$ $\nu^{-1}\left(\left(P_{1}, P_{1}\right)\right),\left\{Q_{1}^{1}, Q_{1}^{2}\right\}=\nu^{-1}\left(\left(Q_{1}, Q_{1}\right)\right)$. Obviously $\mathbb{Z} / 2 \times \mathbb{Z} / 2=\left\{i_{C}, i_{C^{\prime}}\right\}$ acts on $F$ and it interchanges $P_{1}^{1}$ with $P_{1}^{2}$ and $Q_{1}^{1}$ with $Q_{1}^{2}$. In particular $\Gamma=F /\left\langle i_{1} \dot{j}_{1}\right\rangle$ is an elliptic curve, $H^{0}\left(F, \Omega_{F}^{1}\right)=V_{1, \text { id }} \oplus V_{1, \chi_{1}} \oplus V_{1, \chi_{2}}, V_{1, \text { id }} \oplus V_{1, \chi_{1}}=H^{0}\left(C, \Omega_{C}^{1}\right), V_{1, \text { id }} \oplus V_{1, \chi_{2}}=$ $H^{0}\left(C^{\prime}, \Omega_{C^{\prime}}^{1}\right), V_{1, \text { id }}=H^{0}\left(\Gamma, \Omega_{\Gamma}^{1}\right)$. Now consider $\pi_{2}: \widetilde{C} \rightarrow \mathbb{P}^{1}=B$ a double cover branched on $R_{1}, \ldots, R_{2 m}$ points and let $\widetilde{C^{\prime}} \rightarrow B$ be the double cover branched on $A_{1}, A_{2}$. Then the normalization $\widetilde{\nu}: D \rightarrow \widetilde{C} \times{ }_{B} \widetilde{C}^{\prime}$ is a 2-to-1 covering of $\widetilde{C}$ and $g(D)=2 m-1$. If $i_{\widetilde{C}}: \widetilde{C} \rightarrow \widetilde{C}$ and $i_{\widetilde{C}^{\prime}}: \widetilde{C^{\prime}} \rightarrow \widetilde{C^{\prime}}$ are the associated involutions then $\mathbb{Z} / 2 \times \mathbb{Z} / 2=$ $\left\{i_{\widetilde{C}}, i_{\widetilde{C}^{\prime}}\right\}$ acts on $D$ and $\widetilde{\Gamma}=D /\left\langle i_{\widetilde{C}^{\prime}} i_{\widetilde{C}^{\prime}}\right\rangle$ is branched on $R_{1}, \ldots, R_{2 m}, A_{1}, A_{2}$ and it has $g(\widetilde{\Gamma})=m$. Then with the standard notations we have $H^{0}\left(D, \Omega_{D}^{1}\right)=V_{2, \chi_{1}} \oplus V_{2, \chi_{3}}$ where $V_{2, \chi_{1}}=H^{0}\left(\widetilde{C}, \Omega_{\widetilde{C}}^{1}\right), V_{2, \chi_{3}}=H^{0}\left(\widetilde{\Gamma}, \Omega_{\widetilde{\Gamma}}^{1}\right)$. Let $Z=F \times D$, let $G \times Z \rightarrow Z$ be the diagonal action where $G=\left\langle\left(i_{C}, i_{\widetilde{C}}\right)\right\rangle \times\left\langle\left(i_{C^{\prime}}, i_{\widetilde{C}^{\prime}}\right)\right\rangle$ and $X=Z / G$. By the proof of 2.3 we see 
that $H^{0}\left(X, \Omega_{X}^{2}\right) \simeq V_{1, \chi_{1}} \otimes V_{2, \chi_{1}}$ that is $\Phi_{\left|K_{X}\right|}$ is composed with the rational pencil $f_{2}: X \rightarrow B$ and $X$ is in the class $I$ ) of 4.3 . The classes $\left.I I\right)$ and $I I I$ ) can be obtained by a degeneration argument from the class $I$ ). In fact if $R_{1}=A_{1}$ we have $I I$ ) and if $R_{1}=A_{1}, R_{2}=A_{2}$ we have $\left.I I I\right)$.

$\mathbf{q}=\mathbf{b}=\mathbf{1}, \mathbf{g}=\mathbf{3}$ : In 17 Xiao constructed an infinite family of $\mathbb{Z} / 2 \times \mathbb{Z} / 2$-sandwich canonically 3 -fibred surfaces on an elliptic curve. In the following proposition we will classify all the $G$-sandwich canonically 3 -fibred surfaces with $q=b=1, p_{g}(S) \gg 0$. In particular we will show that $a$ ) the family of Xiao is the only $\mathbb{Z} / 2 \times \mathbb{Z} / 2$-infinite family; $b$ ) there is also a $\mathbb{Z} / 2 \times \mathbb{Z} / 2 \times \mathbb{Z} / 2$-infinite family.

Proposition 4.4 If $S$ is a $G$-sandwich canonically 3 -fibred surface on an elliptic curve with $p_{g}(S)=m \geq 2$ then $q(S)=1, S=Z / G$ where $G$ acts diagonally on $Z=F \times D$, $g(F)=3$ and $S$ is in one of the following classes:

Case $G=\mathbb{Z} / 2 \times \mathbb{Z} / 2$ :

$$
\text { I) } g(D)=2 m+1 \quad K_{S}^{2}=8 \chi\left(\mathcal{O}_{S}\right)
$$

Case $G=\mathbb{Z} / 2 \times \mathbb{Z} / 2 \times \mathbb{Z} / 2$ :

$$
\text { II) } g(D)=4 m+1 \quad K_{S}^{2}=8 \chi\left(\mathcal{O}_{S}\right)
$$

Proof. Since $q(S)=1$ and the fibre of the Albanese map is connected, we have that $f_{2}: D \rightarrow B=D / G$ is the Albanese map $g(B)=1$ and $g(A)=0$. By (17) we have to consider all the $G$ actions on $D$ such that the induced decomposition $H^{0}\left(D, \Omega_{D}^{1}\right)=\oplus_{\chi \in G^{\star}} V_{2, \chi}$ satisfies the two conditions in 2.3. We can exclude all the cases with the exceptions of $G=\mathbb{Z} / 2 \times \mathbb{Z} / 2, G=\mathbb{Z} / 2 \times \mathbb{Z} / 2 \times \mathbb{Z} / 2$ as in 4.2 .

The case $G=\mathbb{Z} / 2 \times \mathbb{Z} / 2$.

We consider the system (6) adapted to our hypothesis. By the first $\mathbb{Z} / 2 \times \mathbb{Z} / 2$-action on $F$ we obtain that $\mathcal{L}_{\chi_{1}}, \mathcal{L}_{\chi_{2}}$ and $\mathcal{L}_{\chi_{1} \chi_{2}}$ are non-trivial, torsion line bundles, then $g(D)=1$, i.e. $S$ is not of general type. Let us consider the other $\mathbb{Z} / 2 \times \mathbb{Z} / 2$-action on $F$. ¿From (6) we obtain that $\mathcal{L}_{\chi_{2}}$ is a non-trivial torsion line bundle on the elliptic curve $B$ and $\mathcal{L}_{\chi_{1}}+\mathcal{L}_{\chi_{2}} \equiv \mathcal{L}_{\chi_{1} \chi_{2}}$ where $\operatorname{deg} \mathcal{L}_{\chi_{1}}=m$. Then we have an unique solution and $g(D)=2 m+1$.

The case $G=\mathbb{Z} / 2 \times \mathbb{Z} / 2 \times \mathbb{Z} / 2$.

Since $H^{0}\left(F, \Omega_{F}^{1}\right)=V_{1, \chi_{100}} \oplus V_{1, \chi_{010}} \oplus V_{1, \chi_{001}}$, we can require $V_{2, \chi_{010}}=V_{2, \chi_{001}}=0$ and $\operatorname{dim} V_{2, \chi_{100}}=p_{g}(S)=m>1$. We maintain the notations of the system (10) and it follows that $L_{010}, L_{001}$ and $L_{011}$ are three distinct, non-trivial 2-torsion bundles on the elliptic curve $B$. Moreover $L_{110}=L_{100}+L_{010}, L_{101}=L_{100}+L_{001}, L_{111}=L_{100}+L_{011}$. Then $\operatorname{dim} V_{2, \chi_{100}}=\operatorname{dim} V_{2, \chi_{110}}=\operatorname{dim} V_{2, \chi_{101}}=\operatorname{dim} V_{2, \chi_{111}}=m$ and $g(D)=4 m+1 . \quad \dashv$ 


\subsubsection{Geometrical construction of the two families of proposition 4.4}

The case $G=\mathbb{Z} / 2 \times \mathbb{Z} / 2$ :

The action $G \times F \rightarrow F$ has been described above; see 4.1.1. Let $\widetilde{\pi}: \widetilde{C} \rightarrow B$, $g(B)=1$ be a 2 : 1-cover branched on $R_{1}, \ldots, R_{2 m}$, let $\widetilde{E} \rightarrow B$ a 2-to-1 unramified covering, $g(\widetilde{E})=1$ and we denote by $i_{2}: \widetilde{C} \rightarrow \widetilde{C}, j_{2}: \widetilde{E} \rightarrow \widetilde{E}$ the induced involutions. On the fibre product $\widetilde{E} \times{ }_{B} \widetilde{C}=D$ acts the group $\left\langle i_{2}\right\rangle \times\left\langle j_{2}\right\rangle \equiv$ $\mathbb{Z} / 2 \times \mathbb{Z} / 2$ and $\widetilde{\Gamma}=D /\left\langle i_{2} \dot{j}_{2}\right\rangle$ is an unramified 2-to- 1 cover $\widetilde{\Gamma} \rightarrow B$. We can easily find a basis $\left\langle\alpha_{1}, \ldots \alpha_{m} ; \beta_{1}, \ldots \beta_{m} ; \epsilon\right\rangle=H^{0}\left(D, \Omega_{D}^{1}\right)$ such that $H^{0}\left(\widetilde{C}, \Omega_{\widetilde{C}}^{1}\right) \simeq\left\langle\alpha_{1}, \ldots \alpha_{m} ; \epsilon\right\rangle$, $H^{0}\left(\widetilde{E}, \Omega_{\widetilde{E}}^{1}\right) \simeq\langle\epsilon\rangle \simeq H^{0}\left(\widetilde{\Gamma}, \Omega_{\widetilde{\Gamma}}^{1}\right) \simeq$ and $\left\langle\beta_{1}, \ldots \beta_{m} ; \epsilon\right\rangle$ is also $i_{2}$ anti-invariant . Moreover $V_{2, \chi_{1}}=\left\langle\alpha_{1}, \ldots, \alpha_{m}\right\rangle, V_{2, \chi_{3}}=\left\langle\beta_{1}, \ldots, \beta_{m}\right\rangle, V_{2, \text { id }}=\langle\epsilon\rangle$. Consider $Z=F \times D$ with the $G$-action given by $\langle i, j\rangle$ where $i=\left(i_{1}, i_{2}\right), j=\left(j_{1}, j_{2}\right)$ and let $S=Z / G$. The action is free and since $\left.H^{0}\left(S, \Omega_{S}^{2}\right)=H^{0}\left(Z, \Omega_{Z}^{2}\right)^{G}=\left[\left(V_{1, \chi_{1}} \oplus V_{1, \chi_{2}}\right) \otimes V_{2, \chi_{1}} \oplus V_{2, \chi_{12}}\right)\right]^{G}$ then $\left.H^{0}\left(S, \Omega_{X}^{2}\right)=V_{1, \chi_{1}} \otimes V_{2, \chi_{1}}\right)=\left\{\eta \wedge \alpha_{1}, \ldots, \eta \wedge \alpha_{m}\right\}$; that is $\Phi_{\left|K_{S}\right|}$ yields a pencil with image the elliptic normal curve of degree $m$.

The case $G=\mathbb{Z} / 2 \times \mathbb{Z} / 2 \times \mathbb{Z} / 2$ :

We have constructed the suitable $\mathbb{Z} / 2 \times \mathbb{Z} / 2 \times \mathbb{Z} / 2$-action on the genus-3 curve $F$ in 4.1.1. It is easy to produce the action on $D$. In fact Let $B^{\prime \prime} \rightarrow B$ the 2-to-1 cover of the elliptic curve $B$ branched on $2 m$ points $\left\{A_{i}^{100}\right\}_{i=1, \ldots 2 m}$ and let $B \rightarrow B, B^{\prime \prime} \rightarrow B$ be two distinct $\mathbb{Z} / 2$-unramified covers of $B$. Let $i_{100}: B^{\prime \prime} \rightarrow B^{\prime \prime}, i_{010}: B \rightarrow B, i_{001}: B^{\prime} \rightarrow B^{\prime}$ be the corresponding involutions. If $D=B^{\prime \prime} \times_{B} B \times_{B} B^{\prime}$ then $G=\left\langle i_{100}, i_{010}, i_{001}\right\rangle$ acts on $D$. We set $C_{100}=D /\left\langle i_{100}\right\rangle, C_{010}=D /\left\langle i_{010}\right\rangle, C_{001}=D /\left\langle i_{001}\right\rangle$. Then $C_{100}$ is elliptic, while $g\left(C_{010}\right)=g\left(C_{001}\right)=2 m+1$ and $C_{010} \rightarrow B, C_{001} \rightarrow B$ have branch locus $\left\{A_{i}^{100}\right\}_{i=1, \ldots 2 m}$. It is now easy to see that the decomposition $\oplus_{\chi \in G^{\star}} V_{2, \chi}$ and the diagonal action on the product surface $Z=F \times D$ satisfy 2.3 and they produce the $G$-family of 4.4 .

$\mathbf{q}=\mathbf{2}, \mathbf{b}=\mathbf{0}, \mathbf{g}=\mathbf{3}$ : in [3] [Example 2], Beauville constructed an infinite family of surfaces with $q=2$, canonical map composed with a pencil and non-surjective Albanese map. In 90[Theorem 3.6] Konno showed that this family is essentially unique: that is if $S$ has canonical map composed with a pencil, $q=2, p_{g} \geq 8$ and non-surjective Albanese map then $S$ is the example of Beauville. In the following proposition we give a simple proof of Konno's result in the case of $G$-sandwich canonically fibred surfaces. We remark that we does not assume $g=3$.

Proposition 4.5 If $S$ is a G-sandwich canonically fibred surface with $q(S)=2$, $p_{g}(S)=m \geq 2$ then $S=Z / G$ where $G=\mathbb{Z} / 2$, the group acts diagonally and freely on $Z=F \times D, g(F)=3, g(D)=m, F / G=A$ has genus 2 , the base $D / G=B$ of the canonical fibration is rational and $K_{S}^{2}=8 \chi\left(\mathcal{O}_{S}\right)$.

Proof. Assume that $S$ is a minimal surface with $q(S)=2$ and with canonical fibration $f: S \rightarrow B$ with fibre $F$ of genus $g>1$. Then $K_{S} \equiv Z+f^{\star}(\alpha)$ where $Z, \alpha$ are effective 
divisors on $S$ and $B$ respectively and $h^{0}\left(B, \mathcal{O}_{B}(\alpha)\right)=p_{g}(S)$. By 2.4 $B$ is rational. By the well-known Miyaoka-Yau inequality we have

$$
9\left(p_{g}-1\right) \geq K_{S}^{2}=K_{S} Z+K_{S} f^{\star}(\alpha) \geq(2 g-2)\left(p_{g}-1\right),
$$

then $2 \leq g \leq 5$. In particular if $S$ is a $G$-sandwich canonically fibred surface with top $Z=F \times D$ and base $Z=A \times B$ then $B=B, 3 \leq g(F) \leq 5, g(A)=2$. It is very easy to classify all the Abelian actions with group $G$ such that $F / G=A$ :

\begin{tabular}{|l|c|l|}
\hline$g(F)$ & $G$ & $H^{0}\left(F, \Omega_{F}^{1}\right)$ \\
\hline 3 & $\mathbb{Z} / 2$ & $V_{1, \text { id }} \oplus V_{1, \chi}$ \\
\hline 4 & $\mathbb{Z} / 2$ & $V_{1, \text { id }} \oplus V_{1, \chi}$ \\
\hline 4 & $\mathbb{Z} / 3$ & $V_{1, \text { id }} \oplus V_{1, \chi} \oplus V_{1, \chi^{2}}$ \\
\hline 5 & $\mathbb{Z} / 2$ & $V_{1, \text { id }} \oplus V_{1, \chi}$ \\
\hline 5 & $\mathbb{Z} / 4$ & $V_{1, \text { id }} \oplus V_{1, \chi} \oplus V_{1, \chi^{2}} \oplus V_{1, \chi^{3}}$ \\
\hline 5 & $\mathbb{Z} / 2 \times \mathbb{Z} / 2$ & $V_{1, \text { id }} \oplus V_{1, \chi_{1}} \oplus V_{1, \chi_{2}} \oplus V_{1, \chi_{12}}$ \\
\hline
\end{tabular}

Now we must classify all the $G$ actions on a curve $D$ such that $D / G=B$ and the induced decomposition $H^{0}\left(D, \Omega_{D}^{1}\right)=\oplus_{\chi \in G^{\star}} V_{2, \chi}$ satisfies 2.3. It is an easy computation to show that only the case $g(F)=3$ works and it gives Beauville's family. $\quad-$

We have proved the first theorem stated in the introduction. Now we will show the second one.

\section{$4.2|G|>8$}

The results showed in this section are new. We will prove that there is a rich geometry among the $G$-sandwich canonically 3-fibred surfaces with $|G|>8$. More precisely we will show that only the case $G=\mathbb{Z} / 2 \times \mathbb{Z} / 8$ occurs but it gives many different cases.

Theorem 4.6 If $S$ is a $G$-sandwich canonically 3-fibred surface on a rational curve with $|G|>8$ and $p_{g}(S) \geq 3$ then $q(S)=0, S$ is the minimal desingularization of $X=Z / G$ where $G=\mathbb{Z} / 2 \times \mathbb{Z} / 8$ acts diagonally on $Z=F \times D, g(F)=3$ and $S$ is in one of the following classes:
i) $g(D)=8 m-1-4(a+b) \quad p_{g}(S)=m-a$
$0 \leq a \leq 1,0 \leq b \leq 1$
ii) $g(D)=8 m-4(a+b-1) \quad p_{g}(S)=m+1-(a+b)$
$0 \leq a \leq 2,0 \leq b \leq 1$
iii) $g(D)=8 m+3-4(a+b) \quad p_{g}(S)=m-a$
$0 \leq a \leq 1,0 \leq b \leq 1$
iv) $g(D)=8 m+8-4(a-1) \quad p_{g}(S)=m+1-a$
$0 \leq a \leq 2$

where $a, b, m \in \mathbb{Z}^{+}, m \geq 4$. Moreover the action of $G$ is described completely. 
Proof. We prove the claim by a direct computation. We need to compute all the $G$ actions on a curve $D$ such that $B=D / G$ is a rational curve and the induced decomposition $H^{0}\left(D, \Omega_{D}^{1}\right)=\oplus_{\chi \in G^{\star}} V_{2, \chi}$ satisfies 2.3.

By the standard theory of Abelian covers [see: [11]] we can write the branch locus $\Delta$ of $\rho_{D}: D \rightarrow B$ in the following way: $\Delta=\sum_{H \in \mathcal{C}} \sum_{\psi \in S_{H}} D_{\psi, H}$ where $\mathcal{C}$ is the set of cyclic subgroups of $G, S_{H}$ is the set of generators of $H^{\star}$ for every $H \in \mathcal{C}$ and $D_{\psi, H}$ is the reduced sum of all points $P$ of $D$ such that $H$ is the stabilizer of a(ny) point in $\rho_{D}^{-1}(P)$ and $H$ operates via $\psi$ on the cotangent fibre on $P$.

The case $G=\mathbb{Z} / 9$ :

let $H=\mathbb{Z} / 3$ be the cyclic proper subgroup of $G$ and let $\langle\chi\rangle=G^{\star},\langle\phi\rangle=H^{\star}$. The Galois morphism $\rho_{D}: D \rightarrow B$ is given by a line bundle $L=L_{\chi}$ on $B$ such that:

$$
9 L \equiv D_{\chi}+5 D_{\chi^{2}}+3 D_{\phi}+6 D_{\phi^{2}}+7 D_{\chi^{4}}+2 D_{\chi^{5}}+4 D_{\chi^{7}}+8 D_{\chi^{8}} .
$$

By table (đ) we have to consider the following three cases:

1) $\operatorname{dim} V_{2, \chi^{-1}}=p_{g}(S)$ and $V_{2, \chi^{-2}}=V_{2, \chi^{-4}}=0$;

2) $\operatorname{dim} V_{2, \chi^{-2}}=p_{g}(S)$ and $V_{2, \chi^{-1}}=V_{2, \chi^{-4}}=0$;

3) $\operatorname{dim} V_{2, \chi^{-4}}=p_{g}(S)$ and $V_{2, \chi^{-1}}=V_{2, \chi^{-2}}=0$.

On the other hand all these cases differ by an automorphism of $G$. Then we can compute the first action only: By [11] we have:

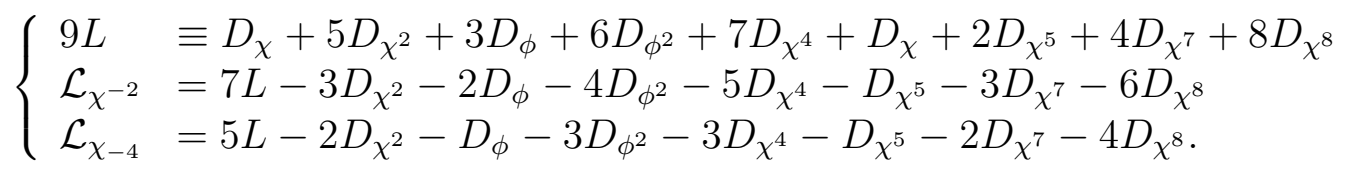

Let $l_{i}=\operatorname{deg} L_{\chi_{i}}, i=1, \ldots, 8, n_{i}=\operatorname{deg} D_{\chi^{i}}$ and $n_{3}=\operatorname{deg} D_{\phi}, n_{6}=\operatorname{deg} D_{\phi^{2}}$. Taking degrees, by 4.2 we obtain:

$$
\left\{\begin{array}{l}
9 l_{1}=n_{1}+5 n_{2}+3 n_{3}+6 n_{6}+7 n_{4}+2 n_{5}+4 n_{7}+8 n_{8} \\
l_{7}=7 l_{1}-\left(3 n_{2}+2 n_{3}+4 n_{6}+5 n_{4}+n_{5}+3 n_{7}+6 n_{8}\right) \\
l_{5}=5 l_{1}-\left(2 n_{2}+n_{3}+3 n_{6}+3 n_{4}+n_{5}+2 n_{7}+4 n_{8}\right)
\end{array}\right.
$$

Since $g(B)=0$ then $l_{7}=l_{5}=1$ and this implies $n_{1}=0, n_{2}=n_{7}=1$ or $n_{2}=0, n_{1}=$ $n_{8}=1$; that is $g(D)=0$ : a contradiction.

The case $G=\mathbb{Z} / 12$ :

let $H_{2}=\mathbb{Z} / 2, H_{3}=\mathbb{Z} / 3, H_{4}=\mathbb{Z} / 4, H_{6}=\mathbb{Z} / 6$ be the cyclic proper subgroups of $G$ and let $\langle\chi\rangle=G^{\star},\left\langle\mu_{i}\right\rangle=H_{i}^{\star}$. To construct $\rho_{D}: D \rightarrow B$ we need a line bundle $L=L_{\chi}$ on $B$ such that:

$$
12 L \equiv D_{\chi}+5 D_{\chi^{5}}+7 D_{\chi^{7}}+11 D_{\chi^{11}}+6 D_{\mu_{2}} 4 D_{\mu_{3}}+8 D_{\mu_{3}^{2}}+3 D_{\mu_{4}}+9 D_{\mu_{4}^{3}}+2 D_{\mu_{6}}+10 D_{\mu_{6}^{5}} .
$$


By 2.3 and table (7) we can assume $\operatorname{deg} L=l=1$, then there are not infinite families. However we can easily find all these surfaces. Set $l_{i}=\operatorname{deg} L_{\chi^{i}}$. The case $l_{5}=1$ and $l_{2} \geq 3$ does not occur; the case $l_{5} \geq 3$ and $l_{2}=1$ has two solutions: $i$ ) $\operatorname{deg} D_{\chi}=7$, $\left.\operatorname{deg} D_{\chi^{5}}=1, D_{\chi^{7}}=D_{\chi^{11}}=D_{\mu_{2}}=D_{\mu_{3}}=D_{\mu_{3}^{2}}=D_{\mu_{4}}=D_{\mu_{4}^{3}}=D_{\mu_{6}}=D_{\mu_{6}^{5}}=0 ; i i\right)$ $\operatorname{deg} D_{\chi}=6, \operatorname{deg} D_{\mu_{2}}=1, \operatorname{deg} D_{\chi^{5}}=D_{\chi^{7}}=D_{\chi^{11}}=D_{\mu_{3}}=D_{\mu_{3}^{2}}=D_{\mu_{4}}=D_{\mu_{4}^{3}}=D_{\mu_{6}}=$ $D_{\mu_{6}^{5}}=0$; both solutions have $p_{g}(S)=2$.

The case $G=\mathbb{Z} / 14$ :

Let $H=\mathbb{Z} / 2, K=\mathbb{Z} / 7$ be the cyclic proper subgroups of $G$ and let $\langle\chi\rangle=G^{\star}$, $\langle\mu\rangle=H^{\star},\langle\psi\rangle=K^{\star}$. To construct $\rho_{D}$ we need a line bundle $L=L_{\chi}$ such that:

$$
14 L \equiv D_{\chi}+5 D_{\chi^{3}}+3 D_{\chi^{5}}+11 D_{\chi^{9}}+9 D_{\chi^{11}}+13 D_{\chi^{13}}+7 D_{\mu}+\sum_{i=1}^{6} 2 i D_{\chi^{2 i}} .
$$

By 2.3 and table (7) we can assume $\operatorname{deg} L=l=1$, then there are not infinite families. Moreover both cases $l_{5}=1$ and $l_{3} \geq 3$ and $l_{5} \geq 3$ and $l_{3}=1$ have solutions, but $p_{g}(S) \leq 2$.

The case $G=\mathbb{Z} / 4 \times \mathbb{Z} / 4$ :

Let $\mathbb{Z} / 4 \simeq H_{10}=\left\langle u \mid u^{4}=1\right\rangle$ and $\mathbb{Z} / 4 \simeq H_{01}=\left\langle v \mid v^{4}=1\right\rangle$. We set $G=H_{10} \times H_{01}$, $H_{11}=\langle u v\rangle, H_{21}=\left\langle u^{2} v\right\rangle, H_{12}=\left\langle u v^{2}\right\rangle, H_{31}=H_{13}=\left\langle v^{3}\right\rangle, T_{10}=\left\langle u^{2}\right\rangle, T_{01}=\left\langle v^{2}\right\rangle$, $T_{11}=\left\langle u^{2} v^{2}\right\rangle$. If $\chi_{1}, \chi_{2}$ generate $(\mathbb{Z} / 4 \times \mathbb{Z} / 4)^{\star}$, the building data of the cover must satisfy:

$$
\left\{\begin{array}{l}
4 \mathcal{L}_{\chi_{1}}=\left[\sum_{i=0}^{3}\left(D_{H_{1 i} \phi_{1 i}}+3 D_{H_{1 i} \phi_{1 i}^{3}}\right)\right]+2\left(D_{H_{21} \phi_{21}}+D_{H_{21} \phi_{21}^{3}}+D_{T_{10}}+D_{T_{11}}\right) \\
4 \mathcal{L}_{\chi_{2}}=\left[\sum_{i=0}^{3}\left(D_{H_{i 1} \phi_{i 1}}+3 D_{H_{i 1} \phi_{i 1}^{3}}\right)\right]+2\left(D_{H_{12} \phi_{12}}+D_{H_{12} \phi_{12}^{3}}+D_{T_{01}}+D_{T_{11}}\right)
\end{array}\right.
$$

By table (7) and by 2.3 we can assume that the action on $D$ has $V_{2, \chi_{2}^{3}}=0$, i.e. $l_{2}=\operatorname{deg} L_{\chi_{2}}=1$. Now we face two cases: $V_{2, \chi_{1}^{3}}=0$ or $V_{2, \chi_{1}^{2} \chi_{2}}=0$. The case $V_{2, \chi_{1}^{2} \chi_{2}}=0$ i.e. $l_{\chi_{1}^{2} \chi_{2}^{3}}=\operatorname{deg} L_{\chi_{1}^{2} \chi_{2}^{3}}=1$ does not occur. If $V_{2, \chi_{1}^{3}}=0$ that is $l_{\chi_{1}}=\operatorname{deg} L_{\chi_{1}}=1$ then $p_{g}(S) \leq 2$.

The case $G=\mathbb{Z} / 2 \times \mathbb{Z} / 8$ :

Let $\mathbb{Z} / 2 \simeq H=\left\langle u \mid u^{2}=1\right\rangle$ and $\mathbb{Z} / 8 \simeq K=\left\langle v \mid v^{8}=1\right\rangle$. We set $G=H \times K$, $H_{2}=\left\langle u v^{4}\right\rangle, K_{2}=\left\langle v^{4}\right\rangle, H_{4}=\left\langle u v^{2}\right\rangle, K_{4}=\left\langle v^{2}\right\rangle, H_{8}=\langle u v\rangle$. If $\chi_{1}, \chi_{2}$ generate $(\mathbb{Z} / 2 \times \mathbb{Z} / 8)^{\star}$, the building data of the cover must satisfy:

$$
\left\{\begin{array}{l}
2 \mathcal{L}_{\chi_{1}}=D_{H}+D_{H_{2}}+D_{H_{4} \phi_{1}}+D_{H_{4} \phi_{1}^{3}}+\sum_{i=1,3,5,7} D_{H_{8} \phi^{i}} \\
8 \mathcal{L}_{\chi_{2}}=4\left(D_{H_{2}}+D_{K_{2}}\right)+2\left(D_{H_{4} \phi_{1}}+K_{H_{4} \psi_{1}}\right)+6\left(D_{H_{4} \phi_{1}^{3}}+D_{K_{4} \psi_{1}^{3}}\right)+\sum_{i=1,3,5,7} i\left(D_{H_{8} \phi^{i}}+D_{K \psi^{i}}\right)
\end{array}\right.
$$

We know that, up to $G$ isomorphisms, the action on $F$ has three branch points on $A$ with the following action: $d_{H_{2}}^{1}=\operatorname{deg} D_{H_{2}}=1, d_{K \psi^{7}}^{1}=\operatorname{deg} D_{K \psi^{7}}=1, d_{H_{8} \phi^{5}}^{1}=$ 
$\operatorname{deg} D_{H_{8} \phi^{5}}=1$. To compute the suitable actions on $D$ we can assume $V_{2, \chi_{2}^{3}}=0$ that is $\operatorname{deg} \mathcal{L}_{\chi_{2}}=1$. Now we have to discuss two cases: $V_{2,\left(\chi_{1} \chi_{2}^{2}\right)^{-1}}=0$ or $V_{2, \chi_{2}^{-3}}=0$.

If $V_{2,\left(\chi_{1} \chi_{2}^{2}\right)^{-1}}=0$ then $\operatorname{deg} \mathcal{L}_{\chi_{1} \chi_{2}^{2}}=1$ and with this condition the system (13) does not have any solution.

If $V_{2, \chi_{2}^{-3}}=0$ then we have to add to (13) the condition $\operatorname{deg} \mathcal{L}_{\chi_{2}^{3}}=1$. We set $\operatorname{deg} \mathcal{L}_{\chi_{1}}=m$ and we find the following solutions:

\begin{tabular}{|l|l|}
\hline$i)$ & Decomposition of $H^{0}\left(D, \Omega_{D}^{1}\right)$ \\
\hline$d_{K \psi^{7}}^{2}=d_{K \psi}^{2}=d_{H_{8} \phi^{7}}^{2}=d_{H_{8} \phi}^{2}=0$ & $l_{\chi_{2}^{i}}^{2}=1, i=1, \ldots, 7$ \\
\hline$d_{H_{4} \phi_{1}}^{2}=d_{H_{4} \phi_{1}^{3}}^{2}=d_{K_{4} \psi_{1}}^{2}=d_{K_{4} \psi_{1}^{3}}^{2}=0$ & $l_{\chi_{1} \chi_{2}}=l_{\chi_{1} \chi_{2}^{3}}=l_{\chi_{1} \chi_{2}^{6}}=m+1-d_{H_{8} \phi^{5}}^{2}$ \\
\hline$d_{H_{2}}^{2}=d_{K_{2}}^{2}=0$ & $l_{\chi_{1} \chi_{2}^{2}}=l_{\chi_{1} \chi_{2}^{5}}=l_{\chi_{1} \chi_{2}^{7}}=m+1-d_{H_{8} \phi^{3}}$ \\
\hline$d_{K \psi^{3}}^{2}+d_{H_{8} \phi^{3}}^{2}=1$ & $l_{\chi_{1} \chi_{2}^{4}}=m+1-d_{H_{8} \phi^{3}}^{2}-d_{H_{8} \phi^{5}}^{2}$ \\
\hline$d_{K \psi^{5}}^{2}+d_{H_{8} \phi^{5}}^{2}=1$ & $g(D)=8 m-1-4\left(d_{H_{8} \phi^{3}}^{2}+d_{H_{8} \phi^{5}}^{2}\right)$ \\
\hline
\end{tabular}

\begin{tabular}{|l|l|}
\hline$i i)$ & Decomposition of $H^{0}\left(D, \Omega_{D}^{1}\right)$ \\
\hline$d_{K \psi^{i}}^{2}=d_{H_{8} \phi^{i}}^{2}=0 i=1,5,7$ & $l_{\chi_{2}^{i}}=1, i=1,3,4,6, l_{\chi_{2}^{j}}=2, j=2,5,7$ \\
\hline$d_{H_{4} \phi_{1}^{3}}^{2}=d_{K_{4} \psi_{1}^{3}}^{2}=0$ & $l_{\chi_{1} \chi_{2}^{2}}=l_{\chi_{1} \chi_{2}^{7}}=m+2-d_{H_{8} \phi^{3}}^{2}-d_{H_{4} \phi_{1}}^{2}$ \\
\hline$d_{H_{2}}^{2}=d_{K_{2}}^{2}=0$ & $l_{\chi_{1} \chi_{2}^{3}}=l_{\chi_{1} \chi_{2}^{6}}=m+1-d_{H_{4} \phi_{1}}, l_{\chi_{1} \chi_{2}}=m+1$ \\
\hline$d_{K \psi^{3}}^{2}+d_{H_{8} \phi^{3}}^{2}=2$ & $l_{\chi_{1} \chi_{2}^{5}}=l_{\chi_{1} \chi_{2}^{4}}=m+1-d_{H_{8} \phi^{3}}^{2}$ \\
\hline$d_{K_{4} \psi_{1}}^{2}+d_{H_{4} \phi_{1}}^{2}=1$ & $g(D)=8 m+4-4\left(d_{H_{8} \phi^{3}}^{2}+d_{H_{4} \phi_{1}}^{2}\right)$ \\
\hline
\end{tabular}

\begin{tabular}{|l|l|}
\hline iii) & Decomposition of $H^{0}\left(D, \Omega_{D}^{1}\right)$ \\
\hline$d_{K \psi^{i}}^{2}=d_{H_{8} \phi^{i}}^{2}=0 i=5,7$ & $l_{\chi_{2}^{i}}=1, i=1,2,3,4,6, l_{\chi_{2}^{j}}=2, j=5,7$, \\
\hline$d_{H_{4} \phi_{1}^{i}}^{2}=d_{K_{4} \psi_{1}^{i}}^{2}=0, i=1,3$ & $l_{\chi_{1} \chi_{2}}=l_{\chi_{1} \chi_{2}^{3}}=m+1-d_{H_{2}}^{2}$ \\
\hline$d_{H_{2}}^{2}+d_{K_{2}}^{2}=1$ & $l_{\chi_{1} \chi_{2}^{2}}=l_{\chi_{1} \chi_{2}^{4}}=m+1-d_{H_{8} \phi^{3}}, l_{\chi_{1} \chi_{2}^{6}}=m+1$ \\
\hline$d_{K \psi^{3}}^{2}+d_{H_{8} \phi^{3}}^{2}=1$ & $l_{\chi_{1} \chi_{2}^{5}}=l_{\chi_{1} \chi_{2}^{7}}=m+2-d_{H_{8} \phi^{3}}-d_{H_{2}}^{2}$ \\
\hline$d_{K \psi}^{2}+d_{H_{8} \phi}^{2}=1$ & $g(D)=8 m+3-4\left(d_{H_{8} \phi^{3}}^{2}+d_{H_{2}}^{2}\right)$ \\
\hline
\end{tabular}

\begin{tabular}{|l|l|}
\hline$i v)$ & Decomposition of $H^{0}\left(D, \Omega_{D}^{1}\right)$ \\
\hline$d_{K \psi^{i}}^{2}=d_{H_{8} \phi^{i}}^{2}=0 i=5,7$ & $l_{\chi_{2}^{1}}=l_{\chi_{2}^{3}} 1, l_{\chi_{2}^{2}}=l_{\chi_{2}^{4}}=l_{\chi_{2}^{6}}=2, l_{\chi_{2}^{5}}=l_{\chi_{2}^{7}}=3$, \\
\hline$d_{H_{4} \phi_{1}^{i}}^{2}=d_{K_{4} \psi_{1}^{i}}^{2}=0, i=1,3$ & $l_{\chi_{1} \chi_{2}}=l_{\chi_{1} \chi_{2}^{3}}=m+1$ \\
\hline$d_{H_{2}}^{2}=d_{K_{2}}^{2}=0$ & $l_{\chi_{1} \chi_{2}^{2}}=l_{\chi_{1} \chi_{2}^{4}}=m+2-d_{H_{8} \phi^{3}}^{2}, l_{\chi_{1} \chi_{2}^{6}}=m+2$ \\
\hline$d_{K \psi^{3}}^{2}+d_{H_{8} \phi^{3}}^{2}=2$ & $l_{\chi_{1} \chi_{2}^{5}}=l_{\chi_{1} \chi_{2}^{7}}=m+3-d_{H_{8} \phi^{3}}$ \\
\hline$d_{K \psi}^{2}+d_{H_{8} \phi}^{2}=2$ & $g(D)=8 m+12-4 d_{H_{8} \phi^{3}}^{2}$ \\
\hline
\end{tabular}

It is an easy task to show that they are the claimed ones. Moreover to obtain the claimed result in table (2) we have to analize the singularities; a straightforward long 
computation. We remark only that in table (2) appears some entries with the same value for $p_{g}$ and $g(D)$, but different $K_{S}^{2}$. In fact this depends only on the type of the singular points.

Theorem 4.7 If $S$ is a $G$-sandwich canonically 3-fibred surface on an elliptic curve with $|G|>8, p_{g}(S)=m \geq 2$ then $q(S)=1, S=Z / G$ where $G=\mathbb{Z} / 2 \times \mathbb{Z} / 8$ acts diagonally and without fixed points on $Z=F \times D, g(F)=3, g(D)=8 m+1$.

Proof. The same argument as for Theorem 4.6, but much more easier. $\dashv$ We have shown the second theorem stated in the introduction; we will explain how to look these new families.

\subsubsection{A geometrical construction of the family of 4.7}

The families in theorem 4.6 have a rich geometry which should be studied. Here we will show the easier geometry of the family in 4.7. We must understand first the $\mathbb{Z} / 2 \times \mathbb{Z} / 8$-action on the genus-3 curve $F$ whose existence is stated in 4.1. We fix three distinct points $Q_{1}, Q_{2}, Q_{3} \in A=\mathbb{P}^{1}$ and we consider the 2-to-1 cover branched on $Q_{2}, Q_{3}, \rho_{\Gamma_{1}}: \Gamma_{1} \rightarrow A$ and the 8-to-1 cover branched on $Q_{1}, Q_{2}, Q_{3}$, $\rho_{\Gamma_{2}}: \Gamma_{2} \rightarrow A$ such that: $\rho_{\Gamma_{1}}^{-1}\left(Q_{2}\right)=Q_{2}^{1}, \rho_{\Gamma_{1}}^{-1}\left(Q_{3}\right)=Q_{3}^{1}, \rho_{\Gamma_{1}}^{-1}\left(Q_{1}\right)=\left\{P_{1}^{1}, P_{2}^{1}\right\}$, $\rho_{\Gamma_{2}}^{-1}\left(Q_{1}\right)=Q_{1}^{2}, \rho_{\Gamma_{2}}^{-1}\left(Q_{3}\right)=Q_{3}^{2}, \rho_{\Gamma_{2}}^{-1}\left(Q_{2}\right)=\left\{P_{1}^{2}, P_{2}^{2}, P_{3}^{2}, P_{4}^{2}\right\}$. If $i_{\Gamma_{1}}: \Gamma_{1} \rightarrow \Gamma_{1}$ and $i_{\Gamma_{2}}: \Gamma_{2} \rightarrow \Gamma_{2}$ and are the generators of the deck-transformation groups of $\rho_{\Gamma_{1}}$ and $\rho_{\Gamma_{2}}$ respectively, then the group $\left\langle i_{\Gamma_{1}}\right\rangle \times\left\langle i_{\Gamma_{2}}\right\rangle \simeq \mathbb{Z} / 2 \times \mathbb{Z} / 8$ acts on the normalization $F$ of the fibre product $\Gamma_{1} \times_{A} \Gamma_{2}, F /\left\langle i_{\Gamma_{1}}\right\rangle=\Gamma_{2}, F /\left\langle i_{\Gamma_{2}}\right\rangle=\Gamma_{1}$. We denote by $\pi_{F}: F \rightarrow A$ the Galois map and we set: $\pi_{F}^{-1}\left(Q_{1}\right)=\left\{A_{1}, A_{2}\right\}, \pi_{F}^{-1}\left(Q_{3}\right)=\left\{B_{1}, B_{2}\right\}$, $\pi_{F}^{-1}\left(Q_{2}\right)=\left\{S_{1}, \ldots, S_{8}\right\}$. Then $G\left(A_{1}\right)=G\left(A_{2}\right)=\left\langle i_{\Gamma_{2}}\right\rangle, G\left(B_{1}\right)=G\left(B_{2}\right)=\left\langle i_{\Gamma_{1}} i_{\Gamma_{2}}\right\rangle$, and $G\left(S_{i}\right)=\left\langle i_{\Gamma_{1}} i_{\Gamma_{2}}^{4}\right\rangle, i=1, \ldots, 8$. It is easy to see that the three canonical divisors: $\operatorname{div} \eta_{1}=2 A_{1}+2 A_{2}, \operatorname{div} \eta_{2}=2 B_{1}+2 B_{2}, \operatorname{div} \eta_{3}=A_{1}+A_{2}+B_{1}+B_{2}$, give a basis $\left\langle\eta_{1}, \eta_{2}, \eta_{3}\right\rangle$ of $H^{0}\left(F, \Omega_{F}^{1}\right)$ such that: $\left\langle\eta_{1}\right\rangle=V_{1, \chi_{2}},\left\langle\eta_{2}\right\rangle=V_{1, \chi_{2}^{3}},\left\langle\eta_{3}\right\rangle=V_{1, \chi_{1} \chi_{2}^{2}}$. On the other hand the $G$-action on $D$ with elliptic quotient $B$ is easily obtained from the fibre product $\widetilde{\Gamma_{1}} \times{ }_{B} \widetilde{\Gamma_{2}}$ where $\widetilde{\Gamma_{1}} \rightarrow B$ is the 2 -to- 1 cover branched on $2 m$ points $R_{1}, \ldots, R_{2 m}$ while $\widetilde{\Gamma_{2}} \rightarrow B$ is an unramified cover of degree 8 . In fact it is easy to obtain a basis $\langle\epsilon\rangle \cup\left\langle\alpha_{i}^{j}\right\rangle_{i=1, \ldots, m}^{j=0, \ldots, 7}$ of $H^{0}\left(F, \Omega_{F}^{1}\right)$ such that: $i_{\widetilde{\Gamma_{1}}}^{\star} \epsilon=\epsilon, i_{\widetilde{\Gamma_{1}}}^{\star} \alpha_{i}^{j}=-\alpha_{i}^{j}$, $i=1, \ldots, m, j=0, \ldots, 7, i_{\widetilde{\Gamma_{2}}}^{\star} \epsilon=\epsilon, i_{\widetilde{\Gamma_{2}}}^{\star} \alpha_{i}^{j}=\mu^{j} \alpha_{i}^{j}, i=1, \ldots, m, j=0, \ldots, 7$ where $\mu$ is a primitive 8-root of the unity. In particular $V_{2 \mathrm{id}}=\langle\epsilon\rangle, V_{2 \chi_{2}^{j}}=0, j=0, \ldots, 7$ and $V_{2 \chi_{1} \chi_{2}^{j}}=\left\langle\alpha_{i}^{8-j}\right\rangle_{j=0, \ldots, 7}, i=1, \ldots, m$. Now it is trivial to see that the $\mathbb{Z} / 2 \times \mathbb{Z} / 8$-diagonal action induced by the group $G=\left\langle\left(i_{\Gamma_{1}} i_{\widetilde{\Gamma_{1}}}\right),\left(i_{\Gamma_{2}} i_{\Gamma_{2}}\right)\right\rangle$ on the product surface $Z=F \times D$ gives a quotient surface $S=Z / G$ such that: $H^{0}\left(X, \Omega_{X}^{1}\right)=H^{0}\left(Z, \Omega_{Z}^{2}\right)^{G} \simeq\langle\epsilon\rangle$ and $H^{0}\left(X, \Omega_{X}^{2}\right)=V_{1, \chi_{1} \chi_{2}^{2}} \otimes V_{2, \chi_{1} \chi_{2}^{6}}=\left\{\eta_{3} \wedge \alpha_{1}^{6}, \ldots, \eta_{3} \wedge \alpha_{m}^{6}\right\}$. In particular $m=p_{g}(S)$ and the image of the canonical map $\Phi_{\left|K_{S}\right|}$ is the normal elliptic curve in $\mathbb{P}^{p_{g}-1}$ of degree $p_{g}$. $\dashv$ 
We end this article with a natural problem: classify all the isotrivial canonical fibrations where the involved group $G$ is not Abelian.

\section{References}

[1] R. Barlow, A simply connected surface of general type with $p_{g}=0$, Invent. Math. 79 (1985), 293-302.

[2] W. Barth, C. Peters, A. Van de Ven, Compact complex surfaces, Ergeb. Math. Grenzgeb. (3) vol 4, Springer Verlag, (1984).

[3] A. Beauville, L'application canonique pour les surfaces de type général, Invent. Math. (1979), 121-140.

[4] E. Brieskorn, Rationale Singularitäten komplexer Flächen, Invent. Math. 4 (1967/68), 336-358.

[5] F. Catanese, An example by F. Zucconi of surfaces $S$ of general type with $q=1$, $p_{g}=g-1$ and $|K|$ yielding $\phi: S \rightarrow V_{g-1}\left(\mathbb{P}^{1}\right)=$ rational normal curve of degree $(g-1)$, with general fibre of $\phi$ a fixed curve $\Gamma^{\prime}$, hyperelliptic of genus 3 , private communication, 1-3.

[6] F. Catanese, Singular Bidouble Cover, preprint (1999).

[7] F. Enriques, Le superficie algebriche, Bologna, Zanichelli (1949).

[8] E. Freitag, Uber die Struktur der Funktionenkörper zu hyperabelschen Gruppen I. J. reine angew. Math 247 (1971), 97-117.

[9] K. Konno, On the irregularity of special non canonical surfaces, Publ. Rims, Kyoto Univ. 30 (1994), 671-688.

[10] M. Miyanishi, J.G. Yang, Surfaces of general type whose canonical map is composed of a pencil of genus 3 with small invariants, J. Math. Kyoto Univ. 38 (1998) no. 1 123-149.

[11] R. Pardini, Abelian Covers, J. reine angew. Math. 417 (1991), 215-219.

[12] H. Pinkham, Singularités rationnelles des surfaces, in Séminaire sur les Singularités des Surfaces Edité par M. Demazure and H. Pinkham and B. Teissier Lectures Notes in Mathematics vol. 777, Springer Verlag (197) 148-172.

[13] G. Pompilj, Alcuni esempi di superficie algebriche a sistema canonico puro degenere, Rend. dei Lincei s. VIII, 4 (1948), 539-544.

[14] F. Serrano, Isotrivial Fibred Surfaces, Annali Math. Pura e Appl. (IV) Vol. CLXXI (1996), 63-81

[15] X. Sun, On Canonical Fibrations of Algebraic Surfaces, Manus. Math. 83, (1994), 161-169 
[16] G. Xiao, Surfaces fibrées en courbes de genre deux, Lect. Notes in Math. vol. 1137, Springer Verlag, (1985).

[17] G. Xiao, L'irrégularité des surfaces de type général dont le systeme canonique est composé d' un pinceau, Comp. Math. 56 (1985), 251-257.

[18] G. Xiao, Birational geometry of algebraic varieties: open problems. The XXIII International Symposium division of Mathematichs. The Taniguchi Foundation Katata, (1988).

[19] F. Zucconi, Numerical inequalities for surfaces with canonical map composed with a pencil, Indag. Mathem. (3), (1998) 459-476.

[20] F. Zucconi, Su alcune questioni relative alle superficie di tipo generale con applicazione canonica composta con un fascio o di grado tre, Tesi di dottorato.

Zucconi Francesco

Università di Udine Dipartimento di Matematica e Informatica

Via delle Scienze 20633100 Udine, Italia

e-mail zucconi@dimi.uniud.it 